\title{
Bihamiltonian structure of the
}

\section{classical superstring in $A d S_{5} \times S^{5}$}

\author{
Andrei Mikhailov \\ California Institute of Technology 452-48, Pasadena CA 91125, USA and \\ Institute for Theoretical and Experimental Physics, 117259, Bol. \\ Cheremushkinskaya, 25, Moscow, Russia
}

\begin{abstract}
We discuss the bihamiltonian structure of the Metsaev-Tseytlin superstring in $A d S_{5} \times S^{5}$. We explicitly write down the boost-invariant symplectic structure for the superstring in $A d S_{5} \times S^{5}$ and explain its relation to the standard (canonical) symplectic structure. We discuss the geometrical meaning of the boost-invariant symplectic structure for the bosonic string.
\end{abstract}

\section{Introduction}

The most well-known example of the AdS/CFT correspondence is the duality between the Type IIB superstring in $A d S_{5} \times S^{5}$ and the $\mathcal{N}=4$ supersymmetric Yang-Mills theory on $\mathbf{R} \times S^{3}$. There is a substantial evidence that both planar $\mathcal{N}=4$ Yang-Mills theory and the string worldsheet theory in $A d S_{5} \times S^{5}$ are quantum integrable systems [1-4]. However at this time there is no satisfactory treatment of the string worldsheet theory as a quantum integrable system. This is partly because of the curved configuration space, but the main reason is perhaps the conformal invariance.

e-print archive: http://lanl.arXiv.org/abs/hep.th/0609108V2 
The technique for dealing with the conformally invariant integrable systems has not been very well developed. And if we gauge fix the conformal invariance by choosing a special set of worldsheet coordinates, such as the light-cone gauge, then we also loose the relativistic invariance.

It turns out that the classical string in $A d S_{5} \times S^{5}$ is closely related to some other integrable systems which do have a relativistic symmetry. A toy example is the relation between the string on $\mathbf{R} \times S^{2}$ and the sine-Gordon model. The sine-Gordon model is a two-dimensional integrable relativistic field theory. On the level of classical equations of motion, the two models are essentially equivalent [5]. The boost symmetries of the sine-Gordon model correspond to some hidden symmetries of the classical string on $\mathbf{R} \times S^{2}$ (to be more precise, these hidden symmetries act on a string modulo the global rotations of $S^{2}$ ). The Poisson structure of the classical string is not invariant under these boosts. But as a classical integrable system, string on $\mathbf{R} \times S^{2}$ has an infinite family of Poisson brackets, which are in some sense mutually compatible. One of these non-standard Poisson brackets is boost-invariant, and in fact coincides with the sine-Gordon symplectic structure.

\subsection{What we will do}

In this paper we will explicitly write down the boost-invariant symplectic structure for the superstring in $A d S_{5} \times S^{5}$ and explain its relation to the standard (canonical) symplectic structure. We first derive the canonical symplectic structure from the string worldsheet action. We then construct some one-parameter group of symmetries of the classical solutions, which is a generalization of the relativistic boosts of the sine-Gordon model. We find that the canonical Poisson structure is not invariant under this oneparameter group of symmetries. However, the canonical Poisson bracket can be written as a sum of three terms:

$$
\{F, G\}^{c a n}=\{F, G\}^{[-2]}+\{F, G\}^{[0]}+\{F, G\}^{[2]},
$$

where the middle term $\{F, G\}^{[0]}$ is boost-invariant, and the terms $\{,\}^{[-2]}$ and $\{,\}^{[2]}$ have scaling degrees -2 and 2 . This means that if $B_{\lambda}$ is the boost transformation with the parameter $\lambda$, then

$$
\left\{B_{\lambda}^{*} F, B_{\lambda}^{*} G\right\}^{[ \pm 2]}=\lambda^{ \pm 2}\{F, G\}^{[ \pm 2]} .
$$

We explicitly verify that the bracket $\{,\}^{[0]}$ satisfies the Jacobi identities. The Jacobi identity for the canonical bracket (1.1) follows from its construction 
as a canonical Poisson bracket. Then equations (1.1) and (1.2) immediately imply that $\{,\}^{[2]}$ and $\{,\}^{[-2]}$ also satisfy the Jacobi identities, and moreover are compatible with $\{,\}^{[0]}$. We then show that $\{,\}^{[-2]}$ can in fact be expressed in terms of $\{,\}^{[0]}$ and $\{,\}^{[2]}$ by equation (5.32). Finally, we give a geometric interpretation of the boost-invariant bracket $\{,\}^{[0]}$ in the purely bosonic case, equation (6.4).

\section{$1.2 \quad$ Earlier work}

Essentially the same results, in the bosonic sector, were previously obtained in [6-15], but from a different perspective. The main difference of our approach is that we start from the relativistic string and derive all the Poisson structures from the canonical Poisson structure of the string worldsheet action. The case of string on $\mathbf{R} \times S^{2}$ was considered in our previous paper [16]. The thorough analysis of the equal-time Poisson brackets in the bosonic sector was presented in [17]; see also $[18,19]$ for an earlier work. In our paper we concentrate on the light-cone Poisson brackets. Also we use the currents of the generalized sine-Gordon model, in order to make the action of the boosts more transparent. The light-cone approach to the non-linear sigma-model was previously used to study Poisson brackets in [20]. Poisson brackets in the pure spinor model were studied in [21].

\subsection{Plan of the paper}

In Section 2 we will review the general definition of the symplectic structure. In Section 3 we will review the nonlinear sigma-model, its relation to the generalized sine-Gordon model, and the hidden relativistic symmetry. Section 4 is a review of the classical superstring in $A d S_{5} \times S^{5}$ and its canonical Poisson structure. In Section 5 we derive the bihamiltonian structure of the classical superstring and discuss its properties. In Section 6 give a geometrical interpretation of the boost-invariant Poisson bracket.

\section{General facts about Poisson brackets and symplectic structure}

Poisson brackets are very important in the classical mechanics, in particular because they are the classical analogue of the quantum mechanical commutators. Poisson brackets are closely related to the symplectic form, and in fact we will use both concepts simultaneously. 


\subsection{Symplectic form}

The symplectic form is computed directly from the classical action, in the following way. Suppose that we have a classical field theory with the action

$$
S=\int d \tau^{+} d \tau^{-} \mathcal{L}[\phi] .
$$

We usually compute the action over the infinite space-time, but let us suppose that we decided to compute the action in a finite region of $\tau^{+}, \tau^{-}$. Let us take $\phi=\phi_{c l}$ a classical solution.

Suppose that we change $\phi_{c l}$ by a small amount $\delta \phi$. We will not require that $\phi_{c l}+\delta \phi$ is again a classical solution, in other words that $\delta \phi$ is "on-shell". But because $\phi_{c l}$ is an extremum of

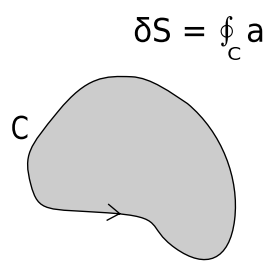
the action, for any $\delta \phi$ we will get: $\delta S=\oint_{C} a$ where $a$ is some 1 -form on the worldsheet. If we restrict $\delta \phi$ to be a classical variation $\delta \phi_{c l}$ of the classical solution, then we get $\delta S$ a 1 -form on the phase space of the system, which is the space of all classical solutions. Since the expression for $a$ contains both $\delta \phi$ and $d \tau$ we can say that $a$ is "a form of the type $(d \tau)(\delta \phi) "$.

Let us restrict $a$ to the tangent space to the space of classical solutions and consider $\omega=\delta a$. This is a form of the type $(d \tau)\left(\delta \phi_{c l}\right)^{2}$, where $\delta \phi_{c l}$ is now onshell. Note that $\omega$ is defined unambiguously, modulo adding a $d$-exact form. This is because $a$ is defined unambiguously modulo a $d$-exact form, because we defined $a$ as a restriction of the unambiguously defined expression with $\delta \phi$ off-shell. Note that $\omega$ is $d$-closed, since $\omega=\delta a$ and $d a$ is $\delta$ of the action density. To define the symplectic form we consider the theory on a cylinder:

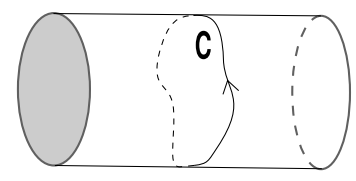

The symplectic form is by definition:

$$
\Omega=\oint_{C} \omega
$$


This is a closed two-form on the phase space. It is also sometimes useful to consider the "symplectic potential" which is defined as $\delta^{-1}$ of the symplectic form:

$$
\alpha=\oint_{C} a .
$$

We have $\delta \alpha=\Omega$ but we have to remember that $\alpha$ depends on the choice of the contour $C$.

For example, consider the particle with the action $\int d t\left(\frac{\dot{x}^{2}}{2}-V(x)\right)$. The phase space can be defined as the space of solutions of the classical equations of motion $\ddot{x}=-V^{\prime}$. The phase space is equipped with the "local" two-form:

$$
\Omega=\delta \dot{x}\left(t_{0}\right) \wedge \delta x\left(t_{0}\right),
$$

which is local in a sense that it requires evaluation of the classical field variables $x$ and $\dot{x}$ at one point in time $t=t_{0}$ and the result of the calculation of $\Omega$ does not depend on the choice of $t_{0}$. In the $d+1$-dimensional classical field theory, with the kinetic term $\int d x^{0} \cdots d x^{d} \partial_{\mu} \Phi \partial^{\mu} \Phi$ we have

$$
\Omega=\int_{C} \delta \Phi \wedge * d \delta \Phi
$$

where $C$ is a $d$-dimensional contour, and $\Omega$ does not depend on the choice of this contour.

\subsection{Poisson bracket}

The Poisson bracket $\{\cdot, \cdot\}$ is defined by a bivector $\theta^{i j}$ which is the inverse of $\Omega_{i j}$ :

$$
\theta^{i k} \Omega_{k j}=\delta_{j}^{i} .
$$

The Poisson bracket of two functions $F$ and $G$ on the phase space is defined by the formula:

$$
\theta=\theta^{i j} \frac{\partial}{\partial \Phi^{i}} \wedge \frac{\partial}{\partial \Phi^{j}} .
$$

The fact that $\Omega$ is closed translates to the Jacobi identity of the Poisson bracket:

$$
\{F,\{G, H\}\}+\{G,\{H, F\}\}+\{H,\{F, G\}\}=0 .
$$

In terms of $\theta$ the Jacobi identity is some bilinear equation:

$$
\llbracket \theta, \theta \rrbracket=0 .
$$




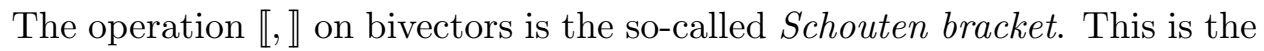
most natural extension of the Lie bracket (the commutator of the vector fields) to the bivectors.

\subsection{The lightcone approach to the description of the symplectic structure}

As we have explained, the symplectic form on the phase space of classical solutions of the two-dimensional field theory is given by $\oint_{C} \omega$ where $\omega$ is something like $\delta \varphi \wedge * d \delta \varphi$, and the integral does not depend on the choice of the contour $C$ because $\omega$ is $d$-closed.

On the string worldsheet, through every point pass two light-like curves (the light cone). We will call the two light-like curves $C^{+}$and $C^{-}$. These curves are called characteristics.

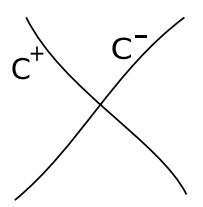

Consider an infinite string worldsheet, and choose the saw-like contour $C$ interpolating between two spacial infinities, consisting of the light-like pieces:

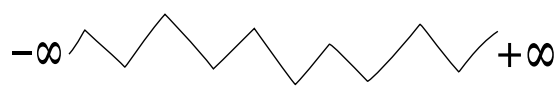

For massive theories, there are excitations which are spacially localized (like breathers of the sine-Gordon model).

If the tooth of the saw is sufficiently large, we can imagine that the intersection of the breather with the contour fits essentially (modulo the exponentially decreasing tails) into one light-like piece. This suggests that the classical solutions rapidly decreasing at infinity can be described in terms of their

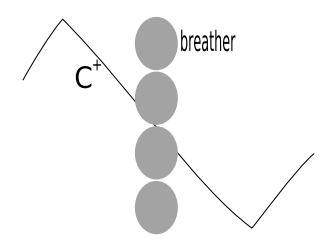
intersection with the characteristic $C^{+}$.

For example, consider the characteristic given by the equation $\tau^{-}=0$. A rapidly decreasing solution $\varphi\left(\tau^{+}, \tau^{-}\right)$is characterized by a function $\varphi\left(\tau^{+}\right)=$ $\varphi\left(\tau^{+}, 0\right)$. The symplectic structure is given by the integral of $\omega$ over the characteristic $C^{+}$:

$$
\Omega=\int_{-\infty}^{+\infty} d \tau^{+} \delta \varphi\left(\tau^{+}\right) \partial_{+} \delta \varphi\left(\tau^{+}\right)
$$


We will call this the "light-cone symplectic structure". There are many subtleties with this approach to describe the symplectic structure (there are important solutions which are not rapidly decreasing at the spacial infinity, see for example [22-24]; if $\varphi\left(\tau^{+}, 0\right)$ goes to zero when $\tau^{+} \rightarrow \pm \infty$, what would guarantee that this is true also at $\tau^{-}=$const $\neq 0$ ?). In this paper we will neglect these subtleties.

\subsection{Compatibility of Poisson brackets}

Two Poisson brackets $\{,\}_{1}$ and $\{,\}_{2}$ are called compatible if their sum $\{,\}_{1}+$ $\{,\}_{2}$ satisfies the Jacobi identity. Integrable systems usually have infinitely many compatible Poisson brackets. Actually, it is enough to have two, and then it is possible to generate an infinite family. Indeed, suppose that the Poisson bivectors $\theta_{1}$ and $\theta_{2}$ give two compatible Poisson brackets. This means that $\llbracket \theta_{1}, \theta_{1} \rrbracket=0$ and $\llbracket \theta_{2}, \theta_{2} \rrbracket=0$ and $\llbracket \theta_{1}, \theta_{2} \rrbracket=0$. Therefore for an arbitrary parameter $t$ the bivector $\theta_{1}+t \theta_{2}$ also satisfies (2.3) and therefore the corresponding two-form $\left(\theta_{1}+t \theta_{2}\right)^{-1}$ is closed. Given that $\left(\theta_{1}\right)^{-1}$ is closed, this implies at small $t$ that $\theta_{1}^{-1} \theta_{2} \theta_{1}^{-1}$ is also closed. This means that $\theta_{1} \theta_{2}^{-1} \theta_{1}$ is again a Poisson bracket.

The expression $N=\theta_{1} \theta_{2}^{-1}=\theta_{1} \Omega_{2}$ is an operator acting on the sections of the tangent bundle to the phase space; in other words, this is a section of $T M \otimes T^{*} M$. Operator $N$ has a special name "recursion operator". The compatibility of the Poisson brackets $\theta_{1}$ and $\theta_{2}$ implies vanishing of the Nijenhuis torsion of $N$, see for example Section 2 of [25] for a concise review.

In this paper the compatible Hamiltonian structures will appear in the following way. We will have some vector field $V$ on the phase space (the infinitesimal boost), and the canonical Poisson bracket will be a sum of three pieces $\theta^{\text {can }}=\theta^{[2]}+\theta^{[0]}+\theta^{[-2]}$, and the action of $V$ on $\theta^{\text {can }}$ (the Lie derivative) will be

$$
V \cdot \theta^{\text {can }}=V \cdot\left(\theta^{[2]}+\theta^{[0]}+\theta^{[-2]}\right)=\theta^{[2]}-\theta^{[-2]} .
$$

Because of the geometrical naturalness of the Lie derivative and the Schouten bracket we have

$$
V \cdot \llbracket \theta_{1}, \theta_{2} \rrbracket=\llbracket V \cdot \theta_{1}, \theta_{2} \rrbracket+\llbracket \theta_{1}, V \cdot \theta_{2} \rrbracket .
$$

Taking into account

$$
\llbracket \theta^{\mathrm{can}}, \theta^{\mathrm{can}} \rrbracket=V \cdot \llbracket \theta^{\mathrm{can}}, \theta^{\mathrm{can}} \rrbracket=\cdots=V^{n} \cdot\left[\theta^{\mathrm{can}}, \theta^{\mathrm{can}} \rrbracket=0\right.
$$


we get

$$
\begin{aligned}
\llbracket \theta^{[2]}, \theta^{[2]} \rrbracket & =0, \\
\llbracket \theta^{[2]}, \theta^{[0]} \rrbracket & =0, \\
\llbracket \theta^{[0]}, \theta^{[0]} \rrbracket+2 \llbracket \theta^{[2]}, \theta^{[-2]} \rrbracket & =0, \\
\llbracket \theta^{[-2]}, \theta^{[0]} \rrbracket & =0, \\
\llbracket \theta^{[-2]}, \theta^{[-2]} \rrbracket & =0 .
\end{aligned}
$$

We will verify explicitly that $\theta^{[0]}$ satisfies the Jacobi identity $\llbracket \theta^{[0]}, \theta^{[0]} \rrbracket=0$. This means that $\theta^{[2]}, \theta^{[0]}$ and $\theta^{[-2]}$ are three mutually compatible Poisson brackets. This way of obtaining compatible Poisson brackets is similar to [26].

\section{Classical string on $\mathrm{R} \times S^{N}$, the non-linear $\sigma$-model and the generalized sine-Gordon system}

\subsection{Classical string and non-linear $\sigma$-model}

Consider the classical string propagating on $\mathbf{R} \times S^{N}$, where the first factor $\mathbf{R}$ is the time, and $S^{N}$ is the space. Let $\mathbf{x}=\left(x^{1}, \ldots, x^{N+1}\right)$ be the unit vector parametrizing $S^{N}$, and let $T$ denote the time (the coordinate parametrizing $\mathbf{R}$ in $\left.\mathbf{R} \times S^{N}\right)$. We introduce on the string worldsheet the special set of coordinates $\left(\tau^{+}, \tau^{-}\right)$which are known as "conformal coordinates". They are characterized by the Virasoro constraint:

$$
\begin{aligned}
& \left(\frac{\partial t}{\partial \tau^{+}}\right)^{2}-\left(\frac{\partial \mathbf{x}}{\partial \tau^{+}}\right)^{2}=0 \\
& \left(\frac{\partial t}{\partial \tau^{-}}\right)^{2}-\left(\frac{\partial \mathbf{x}}{\partial \tau^{-}}\right)^{2}=0
\end{aligned}
$$

Moreover we can choose $\left(\tau^{+}, \tau^{-}\right)$so that

$$
t=\tau^{+}+\tau^{-}
$$

Then we have

$$
\left(\frac{\partial \mathbf{x}}{\partial \tau^{+}}\right)^{2}=\left(\frac{\partial \mathbf{x}}{\partial \tau^{-}}\right)^{2}=1
$$


With these coordinates the equations of motion (the condition that the worldsheet is an extremal surface) become the wave equations:

$$
D_{\overline{0}+} \partial_{-} \mathbf{x}=0
$$

Here $D_{\overline{0}}$ is the standard (Levi-Civita) connection in the tangent space to the sphere:

$$
D_{\overline{0}+} V^{i}=\partial_{+} V^{i}+\Gamma_{j k}^{i} \partial_{+} x^{j} V^{k} .
$$

The index $\overline{0}$ is used for the consistency with the later notations. Equation (3.5) is called the "non-linear sigma-model" (NLSM). The action of the NLSM follows from the Polyakov action of the classical string:

$$
\int d \tau^{+} d \tau^{-}\left(\partial_{+} \mathbf{x}, \partial_{-} \mathbf{x}\right)
$$

The corresponding symplectic structure is

$$
\Omega=\int d \tau^{+}\left(\delta \mathbf{x}, D_{\overline{0}+} \delta \mathbf{x}\right)-\int d \tau^{-}\left(\delta \mathbf{x}, D_{\overline{0}-} \delta \mathbf{x}\right) .
$$

It is convenient to describe the string worldsheet in terms of the group valued function $g\left(\tau^{+}, \tau^{-}\right) \in S O(N+1)$ modulo some gauge symmetry.

Let us pick some (constant) unit vector $\mathbf{x}_{0} \in S^{N}$. Let $g^{-1}$ be the orthogonal matrix which rotates $\mathbf{x}_{0} \in S^{N}$ to $\mathbf{x}\left(\tau^{+}, \tau^{-}\right)$. We have $\mathbf{x}=g^{-1} \mathbf{x}_{0}$. Note that $g$ is defined up to $g \simeq g_{0} g$ where $g_{0} \in$ $S O(N)$. This corresponds to the gauge transformation $g \simeq g_{0} g$. The constant right shift $g \mapsto$ $g C, \quad C \in S O(N+1), C=$ const corresponds to the global rotations of $S^{N}$.

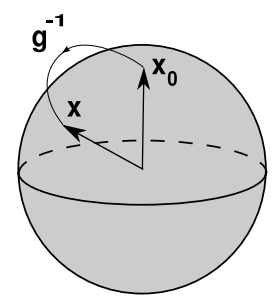

Therefore we can describe the string worldsheet in terms of $g\left(\tau^{+}, \tau^{-}\right)$modulo the gauge invariance

$$
g\left(\tau^{+}, \tau^{-}\right) \equiv g_{0}\left(\tau^{+}, \tau^{-}\right) g\left(\tau^{+}, \tau^{-}\right), \quad g \in S O(N+1), g_{0} \in S O(N) .
$$




\subsection{Classical string and generalized sine-Gordon}

We now want to rewrite the action (3.7) in terms of $g$. Consider the Lie algebras $\mathbf{g}=s o(N+1)$ and $\mathbf{g}_{\overline{0}}=s o(N)$. Introduce the $\mathbf{Z}_{2}$-grading ${ }^{1} \mathbf{g}=$ $\mathbf{g}_{\overline{0}} \oplus \mathbf{g}_{\overline{2}}$ :

$$
\mathbf{g}_{\overline{0}}:\left[\begin{array}{cccccc}
0 & 0 & 0 & 0 & 0 & 0 \\
0 & * & * & * & * & * \\
0 & * & * & * & * & * \\
0 & * & * & * & * & * \\
0 & * & * & * & * & * \\
0 & * & * & * & * & *
\end{array}\right] \quad \mathbf{g}_{\overline{2}}:\left[\begin{array}{cccccc}
0 & * & * & * & * & * \\
* & 0 & 0 & 0 & 0 & 0 \\
* & 0 & 0 & 0 & 0 & 0 \\
* & 0 & 0 & 0 & 0 & 0 \\
* & 0 & 0 & 0 & 0 & 0 \\
* & 0 & 0 & 0 & 0 & 0
\end{array}\right] .
$$

For an element $\xi \in \mathbf{g}$ we introduce the notation

$$
\xi=\xi_{\overline{0}}+\xi_{\overline{2}}, \quad \xi_{\overline{0}} \in \mathbf{g}_{0}, \quad \xi_{\overline{2}} \in \mathbf{g}_{\overline{2}} .
$$

Also introduce the "currents" $J_{ \pm}$:

$$
J_{ \pm}=-\partial_{ \pm} g g^{-1} .
$$

The one-form current is defined as

$$
J=-d g g^{-1}=J_{+} d \tau^{+}+J_{-} d \tau^{-} .
$$

With these notations the action is

$$
S=-\int d \tau^{+} d \tau^{-} \operatorname{tr} J_{\overline{2}_{+}} J_{\overline{2}_{-}} .
$$

The Virasoro constraints are

$$
-\operatorname{tr}\left(J_{\overline{2}_{+}}\right)^{2}=-\operatorname{tr}\left(J_{\overline{2}_{-}}\right)^{2}=1 .
$$

We also have to remember that because of the definition (3.9) the currents satisfy the Maurer-Cartan equation:

$$
d J+J \wedge J=0 .
$$

The symplectic structure is

$$
\Omega=\oint\left[d \tau^{+} \operatorname{tr}\left(\delta g g^{-1}\right)_{\overline{2}} D_{\overline{0}+}\left(\delta g g^{-1}\right)_{\overline{2}}-(+\leftrightarrow-)\right],
$$

\footnotetext{
${ }^{1}$ We use the notation $\mathbf{g}_{\overline{2}}$ rather than $\mathbf{g}_{\overline{1}}$ for the orthogonal complement of $\mathbf{g}_{\overline{0}} \subset \mathbf{g}$, because we want to "leave some space" for the odd generators which will appear in the next section. For the superstring we will have $\mathbf{g}=\mathbf{g}_{\overline{0}} \oplus \mathbf{g}_{\overline{1}} \oplus \mathbf{g}_{\overline{2}} \oplus \mathbf{g}_{\overline{3}}$ where $\mathbf{g}_{\overline{0}} \oplus \mathbf{g}_{\overline{2}}$ will be the even part of the superalgebra and $\mathbf{g}_{\overline{1}} \oplus \mathbf{g}_{\overline{3}}$ the odd part.
} 
where $D_{\overline{0}+}=\partial_{+}+\operatorname{ad}_{\bar{J}_{\overline{0}}}$. The equations of motion are:

$$
\begin{aligned}
\partial_{+} J_{\overline{2}-}+\left[J_{\overline{0}+}, J_{\overline{2}-}\right] & =0, \\
\partial_{-} J_{\overline{2}+}+\left[J_{\overline{0}-}, J_{\overline{2}+}\right] & =0, \\
\partial_{+} J_{\overline{0}_{-}}-\partial_{-} J_{\overline{0}+}+\left[J_{\overline{0}+}, J_{\overline{0}-}\right]+\left[J_{\overline{2}+}, J_{\overline{2}-}\right] & =0 .
\end{aligned}
$$

Note that the equations of motion are written only in terms of $J$. The groupvalued field $g$ is related to $J$ by $J=-d g g^{-1}$, but the equations of motion do not explicitly involve $g$. The "generalized sine-Gordon" is obtained by forgetting about $g$. Consider the space of solutions of the differential equations (3.12), (3.13) and (3.14) with the gauge symmetry

$$
\delta J=d \xi_{0}+\left[J, \xi_{0}\right], \quad \xi_{0} \in \mathbf{g}_{\overline{0}}
$$

and the constraint

$$
\operatorname{tr}\left(J_{\overline{2}+}\right)^{2}=\operatorname{tr}\left(J_{\overline{2}-}\right)^{2}=-1 .
$$

Definition. The system of equations (3.12)-(3.14) with the gauge symmetry (3.15) and the constraint (3.16) is called the generalized sine-Gordon (GSG).

We get the usual sine-Gordon model when $N=2$, for the two-dimensional sphere. In this case we can choose the gauge so that $J$ has the following form:

$$
J_{+}=\left[\begin{array}{ccc}
0 & \cos \varphi & \sin \varphi \\
-\cos \varphi & 0 & -\partial_{+} \varphi \\
-\sin \varphi & \partial_{+} \varphi & 0
\end{array}\right], \quad J_{-}=\left[\begin{array}{ccc}
0 & \cos \varphi & -\sin \varphi \\
-\cos \varphi & 0 & \partial_{-} \varphi \\
\sin \varphi & -\partial_{-} \varphi & 0
\end{array}\right] .
$$

This solves equations (3.12) and (3.13). Equation (3.14) leads to the usual sine-Gordon equation for $\varphi$ :

$$
\partial_{+} \partial_{-} \varphi=-\frac{1}{2} \sin 2 \varphi
$$

In some sense, the generalized sine-Gordon is equivalent to the non-linear $\sigma$-model. One only has to add $g$ satisfying $(d+J) g=0$. But this $g$ is almost defined in terms of $J$, the only ambiguity comes from the integration constants. (Which correspond to $g \mapsto g C, C=$ const, i.e. the global rotations of $S^{N}$.)

The equations of motion of the generalized sine-Gordon can be written as a zero curvature equation if we introduce the spectral parameter $z$. Consider 
the operators:

$$
\begin{aligned}
& \mathcal{L}_{+}[z]=\partial_{+}+J_{\overline{0}+}+\frac{1}{z^{2}} J_{\overline{2}_{+}}, \\
& \mathcal{L}_{-}[z]=\partial_{-}+J_{\overline{0}_{-}}+z^{2} J_{\overline{2}_{-}} .
\end{aligned}
$$

The GSG equations of motion are equivalent to

$$
\left[\mathcal{L}_{+}, \mathcal{L}_{-}\right]=0
$$

In terms of $J$ the symplectic structure equations (3.11) is non-local:

$$
\Omega=\oint d \tau^{+} \operatorname{tr}\left(\left(D_{+}^{-1} \delta J_{+}\right) \overline{2}_{\overline{2}} D_{\overline{0}+}\left(D_{+}^{-1} \delta J_{+}\right) \overline{2}\right)-(+\leftrightarrow-)
$$

But if we add $g$ satisfying $(d+J) g=0$ we get the local formula because

$$
\begin{gathered}
D_{+}^{-1} \delta J_{+}=-\delta g g^{-1} \\
\Omega=\int d \tau^{+} \operatorname{tr}\left(\left(\delta g g^{-1}\right)_{\overline{2}} D_{\overline{0}+}\left(\delta g g^{-1}\right)_{\overline{2}}\right)-(+\leftrightarrow-) .
\end{gathered}
$$

The generalized sine-Gordon itself does not have a local symplectic structure except for the special cases $N=2$ and 3 . To get the local symplectic structure, one has to slightly extend the model by adding finitely many degrees of freedom. For example ${ }^{2}$ adding $g$ satisfying $(d+J) g=0$ we get the canonical symplectic structure of the non-linear $\sigma$-model. In Section 6 we will see that we can add some group-valued fields $g^{L}$ and $g^{R}$ satisfying (6.3) and get the non-standard symplectic structure (6.4).

\footnotetext{
${ }^{2}$ Another possibility would be to add the g-valued field $\Psi$ satisfying $D \Psi=* J_{\overline{2}}$. We would then get the symplectic structure: $\Omega=\oint \delta \Psi \delta J$. This corresponds to the action $S=\int \operatorname{tr}\left(\Psi\left(d J+J^{2}\right)+J_{\overline{2}} \wedge * J_{\overline{2}}\right)$ The equation of motion for $\Psi$ implies the existence of $g$ such that $J=-d g g^{-1}$ and the action on-shell is equal to the standard action $\int d \tau^{+} d \tau^{-} \operatorname{tr}\left(\left(\partial_{+} g g^{-1}\right)_{\overline{2}}\left(\partial_{-} g g^{-1}\right)_{\overline{2}}\right)$ and therefore gives essentially the same symplectic structure, modulo subtleties with boundary conditions. Note that the expression for $g$ in terms of $J$ is non-local, so it is strictly speaking a different model. It could be thought of as a "T-dual" of the classical string $[27,28]$.
} 


\subsection{Relativistic symmetry}

There is an obvious symmetry under the constant shifts of $\tau^{+}$and $\tau^{-}$. But besides shifts, the GSG equations are also symmetric under boosts:

$$
\begin{aligned}
& J_{\overline{0}_{ \pm}}\left(\tau^{+}, \tau^{-}\right) \mapsto \lambda^{ \pm 1} J_{\overline{0} \pm}\left(\lambda \tau^{+}, \lambda^{-1} \tau^{-}\right), \\
& J_{\overline{2}_{ \pm}}\left(\tau^{+}, \tau^{-}\right) \mapsto J_{\overline{2} \pm}\left(\lambda \tau^{+}, \lambda^{-1} \tau^{-}\right) .
\end{aligned}
$$

This can be thought of as the rescaling of $\left(\tau_{+}, \tau_{-}\right) \mapsto\left(\lambda \tau^{+}, \lambda^{-1} \tau^{-}\right)$combined with the rescaling of the spectral parameter $z \mapsto \lambda^{-1 / 2} z$.

We will use this relativistic symmetry to introduce the bihamiltonian structure of the GSG. It turns out that the canonical Poisson structure of the NLSM leads to the Poisson structure $\theta^{\text {str }}$ of the GSG which is not invariant under the relativistic symmetry. More precisely we will have:

$$
\theta_{\text {str }}=\theta^{[2]}+\theta^{[0]}+\theta^{[-2]}
$$

where $\theta^{[0]}$ is invariant under the boosts, and $\theta^{[2]}$ and $\theta^{[-2]}$ transform as $\lambda^{2}$ and $\lambda^{-2}$, respectively. We will explicitly verify that $\theta^{[0]}$ satisfies the Jacobi identities. Then the arguments of Section 2.4 imply that $\theta^{[2]}$ and $\theta^{[-2]}$ also satisfy the Jacobi identities, and moreover all three brackets $\theta^{[0]}, \theta^{[2]}$ and $\theta^{[-2]}$ are mutually compatible. We will also find that $\theta^{[-2]}$ is related to $\theta^{[0]}$ and $\theta^{[2]}$ by equation (5.32).

\section{Superstring in $A d S_{5} \times S^{5}$}

In this section we will use a variant of the Metsaev-Tseytlin description of the superstring in $A d S_{5} \times S^{5}[29]$.

\subsection{The superalgebra $\mathrm{g}=p s u(2,2 \mid 4)$}

One of the most important properties of this superalgebra is the existence of a $\mathbf{Z}_{4}$ grading [29-32]. There are many ways to explain this grading. For example, we can use the correspondence between the bosonic generators of the superalgebra and the Killing vector fields on $A d S_{5} \times S^{5}$. The fermionic generators correspond to Killing spinors. We can embed $A d S_{5} \times S^{5}$ into the flat space $\mathbf{R}^{2+10}$ as the direct product of the hyperboloid and the sphere. As explained in [33,34], the Killing spinors in $A d S_{5} \times S^{5}$ correspond to the constant spinors in $\mathbf{R}^{2+10}$ satisfying some chirality condition. The spinor 
bundle of $A d S_{5} \times S^{5}$ is naturally identified with the subbundle of the spinor bundle of $\mathbf{R}^{2+10}$, which is the image of the projector

$$
\frac{1}{2}\left(1+\Gamma\left(e_{A}^{\perp}\right) \Gamma\left(e_{S}^{\perp}\right)\right)
$$

Here $e_{S}^{\frac{1}{S}}$ is the vector field normal to the surface of $S^{5}$ in $\mathbf{R}^{6}$, and $e_{A}^{\perp}$ is normal to the surface of the hyperboloid in $\mathbf{R}^{2+4}$. For any vector $v$ we denote $\Gamma(v)$ the corresponding $\Gamma$-matrix $\Gamma_{\mu} v^{\mu}$. We assume that $\Gamma_{-1}^{2}=\Gamma_{0}^{2}=1$ and $\Gamma_{1}^{2}=\ldots=\Gamma_{10}^{2}=-1$. The covariantly constant spinors corresponds to the sections of the form

$$
\psi=\frac{1}{2}\left(1+\Gamma\left(e_{A}^{\perp}\right) \Gamma\left(e_{S}^{\perp}\right)\right) \Psi_{++}
$$

where $\Psi_{++}$is a constant spinor with the chirality conditions:

$$
\begin{aligned}
\Gamma_{-1} \Gamma_{0} \Gamma_{1} \Gamma_{2} \Gamma_{3} \Gamma_{4} \Psi_{++} & =i \Psi_{++} \\
\Gamma_{5} \Gamma_{6} \Gamma_{7} \Gamma_{8} \Gamma_{9} \Gamma_{10} \Psi_{++} & =i \Psi_{++}
\end{aligned}
$$

In this situation we will write

$$
\Psi_{++}=\mathcal{S}(\psi)
$$

We defined $\mathcal{S}$ as the map (4.1) from the spinors in $A d S_{5} \times S^{5}$ to the spinors in $\mathbf{R}^{2+10}$ with the chirality conditions (4.2) and (4.3). The $\mathbf{Z}_{4}$ grading depends on the choice of a point $x_{0} \in A d S_{5} \times S^{5}$. Let $e_{0 A}^{\perp}$ and $e_{0 S}^{\perp}$ be the corresponding unit normals at the point $x_{0}$. The $\mathbf{Z}_{4}$ grading is defined by the operator $\Lambda$ :

$$
\Lambda \Psi_{++}=\Gamma\left(e_{0 A}^{\perp}\right) \Gamma\left(e_{0 S}^{\perp}\right) \Psi_{++}^{*} .
$$

Note that $\Lambda^{2}=\mathbf{1}$. We will say that $\psi$ given by (4.1) belongs to $\mathbf{g}_{\overline{1}}$ if $\Lambda \Psi_{++}=$ $\Psi_{++}$and to $\mathbf{g}_{\overline{3}}$ if $\Lambda \Psi_{++}=-\Psi_{++}$. Here $*$ means complex conjugation, and we use such a representation of the $\Gamma$-matrices $\Gamma_{-1}, \ldots, \Gamma_{10}$ that all their components are real numbers. As in the bosonic case, $\mathbf{g}_{\overline{0}}$ is the stabilizer of $x_{0}$ and $\mathbf{g}_{\overline{2}}$ is the bosonic part of the orthogonal complement to this stabilizer.

\subsection{Classical action and the canonical symplectic structure}

For the superstring the current $J$ belongs to the superalgebra $\mathbf{g}=p s u(2,2 \mid 4)$ and can be decomposed according to its $\mathbf{Z}_{4}$ grading:

$$
J=J_{\overline{0}}+J_{\overline{1}}+J_{\overline{2}}+J_{\overline{3}} .
$$

(For the purely bosonic string we had only the even components $J_{\overline{0}}$ and $J_{\overline{2}}$.) 
The classical action is

$$
S=\frac{1}{2} \iint \operatorname{str}\left[J_{\overline{2}} * J_{\overline{2}}+J_{\overline{1}} J_{\overline{3}}\right] .
$$

The Maurer-Cartan equations are:

$$
\begin{aligned}
d J_{2}+J_{0} J_{2}+J_{2} J_{0}+J_{1}^{2}+J_{3}^{2} & =0, \\
d J_{1}+J_{0} J_{1}+J_{1} J_{0}+J_{2} J_{3}+J_{3} J_{2} & =0, \\
d J_{3}+J_{0} J_{3}+J_{3} J_{0}+J_{1} J_{2}+J_{2} J_{1} & =0 .
\end{aligned}
$$

The equations of motion are:

$$
\begin{aligned}
d * J_{2}+J_{0} * J_{2}+* J_{2} J_{0}-J_{1}^{2}+J_{3}^{2} & =0, \\
{\left[J_{3-}, J_{2+}\right] } & =0, \\
{\left[J_{1+}, J_{2-}\right] } & =0 .
\end{aligned}
$$

The symplectic potential (2.1) follows from the on-shell variation of the action:

$$
\alpha=\frac{1}{2} \int \operatorname{str}\left(2\left(\delta g g^{-1}\right)_{\overline{2}} * J_{\overline{2}}+\left(\delta g g^{-1}\right)_{\overline{1}} J_{\overline{3}}-\left(\delta g g^{-1}\right)_{\overline{3}} J_{\overline{1}}\right) .
$$

The symplectic form is $\Omega=\delta \alpha$ :

$$
\begin{aligned}
\Omega= & \int \operatorname{str}\left\{-\left(\delta g g^{-1}\right)_{\overline{2}} \wedge * D_{J_{\overline{0}}}\left(\delta g g^{-1}\right)_{\overline{2}}\right. \\
& +\left(J_{\overline{2}}-* J_{\overline{2}}\right)\left(\delta g g^{-1}\right)_{\overline{1}} \wedge\left(\delta g g^{-1}\right)_{\overline{1}} \\
& -\left(J_{\overline{2}}+* J_{\overline{2}}\right)\left(\delta g g^{-1}\right)_{\overline{3}} \wedge\left(\delta g g^{-1}\right)_{\overline{3}} \\
& +\left(J_{\overline{1}}+* J_{\overline{1}}\right)\left(\left(\delta g g^{-1}\right)_{\overline{1}} \wedge\left(\delta g g^{-1}\right)_{\overline{2}}+\left(\delta g g^{-1}\right)_{\overline{2}} \wedge\left(\delta g g^{-1}\right)_{\overline{1}}\right) \\
& \left.-\left(J_{\overline{3}}-* J_{\overline{3}}\right)\left(\left(\delta g g^{-1}\right)_{\overline{3}} \wedge\left(\delta g g^{-1}\right)_{\overline{2}}+\left(\delta g g^{-1}\right)_{\overline{2}} \wedge\left(\delta g g^{-1}\right)_{\overline{3}}\right)\right\} .
\end{aligned}
$$

This form is strictly speaking not symplectic, because the variations

$$
\delta g g^{-1}=f^{+}\left(\tau^{+}, \tau^{-}\right) J_{\overline{1}+}+f^{-}\left(\tau^{+}, \tau^{-}\right) J_{\overline{3}-}
$$

are in the kernel of $\Omega$ for an arbitrary $f^{ \pm}\left(\tau^{+}, \tau^{-}\right)$. The symplectic form by definition should be non-degenerate; we should have called $\Omega$ "presymplectic". The variation (4.10) should therefore be considered a gauge transformation. It preserves the equations of motion.

The situation in flat space is similar, but technically simpler. There are two fermions $\theta^{1}$ and $\theta^{2}$. Let us restrict ourselves with the quadratic order, in the 
fermions. The currents $J_{\overline{1}}$ and $J_{\overline{3}}$ correspond to $d \theta^{1}$ and $d \theta^{2}$. The equations of motion $\left[J_{\overline{2}_{+}}, J_{\overline{3}_{-}}\right]=0$ and $\left[J_{\overline{2}_{-}}, J_{\overline{1}_{+}}\right]=0$ correspond to

$$
\widehat{\partial_{+} x} \partial_{-} \theta^{2}=\widehat{\partial_{-} x} \partial_{+} \theta^{1}=0 \text {. }
$$

The symplectic form (assuming $\widehat{\partial_{+} x}$ and $\widehat{\partial_{-} x}$ constant) is

$$
\Omega=\int d \tau^{-} \delta \overline{\theta^{1}} \widehat{\partial_{-} x} \delta \theta^{1}+\int d \tau^{+} \delta \overline{\theta^{2}} \widehat{\partial_{+} x} \delta \theta^{2} .
$$

The kernel is $\delta_{f} \theta^{1}=f^{+} \partial_{+} \theta^{1}$ and $\delta_{f} \theta^{2}=f^{-} \partial_{-} \theta^{2}$ (to verify that this is in the kernel of (4.12), we have to use (4.11)). But this is actually a kappasymmetry. There are such $\kappa^{1}$ and $\kappa^{2}$ that $\delta_{f} \theta^{1}=\widehat{\partial_{-}} x \kappa^{1}$ and $\delta_{f} \theta^{2}=\widehat{\partial_{+} x} \kappa^{2}$. On the other hand, there is a gauge with $\partial_{+} \theta^{1}=\partial_{-} \theta^{2}=0$. (We used this gauge in [35].)

We will put

$$
J_{\overline{1}+}=J_{\overline{3}-}=0 .
$$

This gives a gauge-fixed version of the Metsaev-Tseytlin formalism. The equations of motion become

$$
\begin{aligned}
& D_{\overline{0}+} J_{\overline{2}_{-}}=D_{\overline{0}-} J_{\overline{2}+}=0, \\
& \left(D_{+} J_{-}\right)_{\overline{1}}=\left(D_{-} J_{+}\right)_{\overline{3}}=0 .
\end{aligned}
$$

This can be understood as the consistency condition for the zero curvature equation:

$$
\left[D_{\overline{0}+}+\frac{1}{z} J_{\overline{3}+}+\frac{1}{z^{2}} J_{\overline{2}+}, D_{0-}+z J_{\overline{1}-}+z^{2} J_{\overline{2}-}\right]=0 .
$$

Equation (4.13) implies the following constraint on $\xi=\delta g g^{-1}$ :

$$
\begin{aligned}
D_{\overline{0}+} \xi_{\overline{1}}+\left[J_{\overline{2}+}, \xi_{\overline{3}}\right]+\left[J_{\overline{3}_{+}}, \xi_{\overline{2}}\right] & =0, \\
D_{\overline{0}-} \xi_{\overline{3}}+\left[J_{\overline{2}-}, \xi_{\overline{1}}\right]+\left[J_{\overline{1}-}, \xi_{\overline{2}}\right] & =0 .
\end{aligned}
$$

In the classical string worldsheet theory $J$ should satisfy the Virasoro constraint:

$$
\operatorname{str} J_{\overline{2}+}^{2}=0 \text {. }
$$

In what follows we will assume that the Virasoro constraints are satisfied. 


\subsection{Gauge transformations and dressing transformations}

Let us consider the left invariant vector fields $L_{\xi}$ such that $L_{\xi} \cdot g=-\xi g$. The symplectic form is

$$
\begin{aligned}
\Omega\left(L_{\xi}, L_{\eta}\right)= & \int d \tau^{+} \operatorname{str}\left(\eta_{\overline{2}} \stackrel{\leftrightarrow}{D}_{\overline{0}+} \xi_{\overline{2}}-\eta_{\overline{3}} \stackrel{\leftrightarrow}{a d_{J_{\overline{2}}+}} \xi_{\overline{3}}\right) \\
& -\int d \tau^{-} \operatorname{str}\left(\eta_{\overline{2}} \stackrel{\leftrightarrow}{D}_{\overline{0}-} \xi_{\overline{2}}-\eta_{\overline{1}} \overleftrightarrow{a d}_{J_{\overline{2}-}} \xi_{\overline{1}}\right)
\end{aligned}
$$

Strictly speaking, this is not yet a symplectic form, because it still has a kernel. The kernel is generated by the vectors of the form $\left(\xi_{\overline{0}}, \xi_{\overline{1}}, \xi_{\overline{2}}, \xi_{\overline{3}}\right)=$ $\left(0,0,0, \chi_{\overline{3}}\right)$ where $\chi_{\overline{3}}$ is such that $\left[J_{\overline{2}+}, \chi_{\overline{3}}\right]=0$ and $D_{\overline{0}-} \chi_{\overline{3}}=0$, and the vectors of the form $\left(\xi_{\overline{0}}, \xi_{\overline{1}}, \xi_{\overline{2}}, \xi_{\overline{3}}\right)=\left(0, \chi_{\overline{1}}, 0,0\right)$ where $\chi_{\overline{1}}$ is such that $\left[J_{\overline{2}-}, \chi_{\overline{1}}\right]=$ 0 and $D_{\overline{0}+} \chi_{\overline{1}}=0$. These are the residual gauge transformations. These gauge transformations can be considered as particular dressing transformations with the parameter $\chi(z)=z^{-1} \chi_{\overline{3}}+z \chi_{\overline{1}}$ :

$$
\begin{aligned}
\delta J(z) & =[d+J(z), \chi(z)], \\
\chi(z) & =z^{-1} \chi_{\overline{3}}+z \chi_{\overline{1}}, \\
{\left[J_{\overline{2}-}, \chi_{\overline{1}}\right] } & =0, \quad D_{\overline{0}_{+} \chi_{\overline{1}}=0,} \\
{\left[J_{\overline{2}+}, \chi_{\overline{3}}\right] } & =0, \quad D_{\overline{0}_{-}} \chi_{\overline{3}}=0 .
\end{aligned}
$$

In the lightcone formalism, the independent variables are $J_{+}$. We will fix these residual gauge transformations by requiring that there exists $K_{\overline{1}}$ such that

$$
J_{\overline{3}+}=\left[J_{\overline{2}+}, K_{\overline{1}}\right] .
$$

In terms of the 12-dimensional spinors:

$$
\mathcal{S}\left(\hat{J}_{\overline{3}+}\right)=\left({\widehat{\partial_{+} x}}_{S} \Gamma\left(e_{S}^{\perp}\right)+{\widehat{\partial_{+} x}}_{A} \Gamma\left(e_{A}^{\perp}\right)\right) \mathcal{S}\left(K_{\overline{1}}\right) .
$$

We have to explain why (4.26) is a reasonable gauge choice. Consider the projection operator

$$
\mathcal{P}=\frac{1}{2}\left(1-{\widehat{\partial_{+} x}}_{S} \Gamma\left(e_{S}^{\frac{1}{S}}\right){\widehat{\partial_{+} x}}_{A} \Gamma\left(e_{A}^{\perp}\right)\right) .
$$

Note that $\mathcal{P}^{2}=\mathbf{1}$. We have:

$$
\begin{aligned}
\operatorname{Ker}\left(\operatorname{ad}_{J_{\overline{2}+}}: \mathbf{g}_{\overline{3}} \rightarrow \mathbf{g}_{\overline{1}}\right) & =\operatorname{Ker} \mathcal{P} \\
\operatorname{Im}\left(\operatorname{ad}_{J_{\overline{2}+}}: \mathbf{g}_{\overline{1}} \rightarrow \mathbf{g}_{\overline{3}}\right) & =\operatorname{Im} \mathcal{P} .
\end{aligned}
$$


Now suppose that

$$
J_{\overline{3}+}=\left[J_{\overline{2}+}, K_{\overline{1}}\right]+\Delta J_{\overline{3}+},
$$

where $\Delta J_{\overline{3}_{+}}$is small and belongs to $\operatorname{Ker}\left(\operatorname{ad}_{J_{\overline{2}}+}: \mathbf{g}_{\overline{3}} \rightarrow \mathbf{g}_{\overline{1}}\right)$. We want to prove that there is a small $\chi_{\overline{3}} \in \operatorname{Ker}\left(\operatorname{ad}_{\bar{J}_{\overline{2}+}}: \mathbf{g}_{\overline{3}} \rightarrow \mathbf{g}_{\overline{1}}\right)$ and $\Delta K_{\overline{1}}$ such that

$$
D_{\overline{0}+} \chi_{\overline{3}}-\operatorname{ad}_{\overline{\overline{2}}_{+}} \Delta K_{\overline{1}}=\Delta J_{\overline{3}+} \cdot
$$

This means that we are looking for $\chi_{\overline{3}}$ such that

$$
(1-\mathcal{P}) D_{\overline{0}+} \chi_{\overline{3}}=\Delta J_{\overline{3}+} .
$$

Therefore we have to prove that the operator

$$
\mathcal{A}=(1-\mathcal{P}) D_{\overline{0}_{+}}: \operatorname{Ker} \mathcal{P} \rightarrow \operatorname{Ker} \mathcal{P}
$$

is invertible. This is true when $J_{\overline{0}+}=0$ and $J_{\overline{2}+}=$ const because in this case we have $\left.(1-\mathcal{P}) D_{\overline{0}+}\right|_{\operatorname{Ker} \mathcal{P}}=\partial_{+}$. This means that $\mathcal{A}$ will remain invertible at least for small enough $J_{\overline{0}+}$ and slowly varying $J_{\overline{2}+}$.

\section{Bihamiltonian structure of the classical superstring}

\subsection{Hidden relativistic symmetry}

The relativistic symmetry acts in the following way:

$$
\begin{aligned}
& J_{\overline{0}_{ \pm}}\left(\tau^{+}, \tau^{-}\right) \mapsto \lambda^{ \pm 1} J_{\overline{0}_{ \pm}}\left(\lambda \tau^{+}, \lambda^{-1} \tau^{-}\right), \\
& J_{\overline{1}-}\left(\tau^{+}, \tau^{-}\right) \mapsto \lambda^{-1 / 2} J_{\overline{1}_{-}}\left(\lambda \tau^{+}, \lambda^{-1} \tau^{-}\right), \\
& J_{\overline{3}_{+}}\left(\tau^{+}, \tau^{-}\right) \mapsto \lambda^{1 / 2} J_{\overline{3}+}\left(\lambda \tau^{+}, \lambda^{-1} \tau^{-}\right), \\
& J_{\overline{2} \pm}\left(\tau^{+}, \tau^{-}\right) \mapsto J_{\overline{2} \pm}\left(\lambda \tau^{+}, \lambda^{-1} \tau^{-}\right) .
\end{aligned}
$$

This is a symmetry of the equations of motion (4.14) and (4.15). This can be thought of as the rescaling of $\left(\tau_{+}, \tau_{-}\right) \mapsto\left(\lambda \tau^{+}, \lambda^{-1} \tau^{-}\right)$combined with the rescaling of the spectral parameter $z \mapsto \lambda^{-1 / 2} z$.

\subsection{The canonical Poisson bracket}

In this section we will calculate the Poisson bracket corresponding to the symplectic form (4.20) and (4.21). We use the lightcone formalism, so only 
the first line (4.20) is important for us. The integral over $d \tau^{+}$is from $-\infty$ to $+\infty$, at constant $\tau^{-}$.

Let us formally resolve the constraint (4.17):

$$
\xi_{\overline{1}}=-D_{\overline{0}+}^{-1}\left(\left[J_{\overline{2}+}, \xi_{\overline{3}}\right]+\left[J_{\overline{3}+}, \xi_{\overline{2}}\right]\right) .
$$

Consider the variation with $\xi_{\overline{0}}=0$ and $\xi_{\overline{1}}$ given by (5.2):

$$
\begin{aligned}
& \delta J_{\overline{3}+}=D_{\overline{0}+} \xi_{\overline{3}}-\left[J_{\overline{2}+}, D_{\overline{0}+}^{-1}\left(\left[J_{\overline{2}+}, \xi_{\overline{3}}\right]+\left[J_{\overline{3}+}, \xi_{\overline{2}}\right]\right)\right], \\
& \delta J_{\overline{2}+}=D_{\overline{0}+} \xi_{\overline{2}}+\left[J_{\overline{3}+}, \xi_{\overline{3}}\right], \\
& \delta J_{\overline{0}+}=\left[J_{\overline{2}+}, \xi_{\overline{2}}\right]-\left[J_{\overline{3}+}, D_{\overline{0}+}^{-1}\left(\left[J_{\overline{2}+}, \xi_{\overline{3}}\right]+\left[J_{3+}, \xi_{\overline{2}}\right]\right)\right] .
\end{aligned}
$$

Consider a functional $F$, which is gauge invariant under $\delta J=\left[d+J, \xi_{\overline{0}}\right]$ and under (4.22). Being invariant under (4.22) implies that

$$
\begin{aligned}
& \operatorname{ad}_{J_{\overline{3}}+} \frac{\delta F}{\delta J_{\overline{2}+}}+D_{\overline{0}+} \frac{\delta F}{\delta J_{\overline{3}+}} \in \operatorname{Im}\left(\operatorname{ad}_{J_{\overline{2}}}\right), \\
& \operatorname{ad}_{J_{\overline{3}+}} \frac{\delta F}{\delta J_{\overline{0}+}}+\operatorname{ad}_{J_{\overline{2}}+} \frac{\delta F}{\delta J_{\overline{3}+}} \in \operatorname{Im}\left(D_{\overline{0}+}\right) .
\end{aligned}
$$

Let us find $\eta$ such that the $L_{\eta}$ is the Hamiltonian vector field generated by F:

$$
\Omega\left(L_{\eta}, L_{\xi}\right)=L_{\xi} . F, \quad \text { for any } \xi .
$$

Let us denote $a_{\overline{2}}=\operatorname{ad}_{J_{\overline{2}+}}$ and $a_{\overline{3}}=\operatorname{ad}_{\bar{J}_{\overline{3}}}$. A straightforward calculation gives:

$$
\begin{aligned}
\eta_{\overline{1}}= & \frac{\delta F}{\delta J_{\overline{3}+}}+\left(\left(D_{\overline{0}+}^{-1} a_{\overline{3}}\right)^{2} D_{\overline{0}+}^{-1} a_{\overline{2}}-\left(D_{\overline{0}+}^{-1} a_{\overline{2}}\right)^{2}\right) \frac{\delta F}{\delta J_{\overline{3}+}} \\
& -\left(D_{\overline{0}+}^{-1} a_{\overline{2}} D_{\overline{0}+}^{-1} a_{\overline{3}}+D_{\overline{0}+}^{-1} a_{\overline{3}} D_{\overline{0}+}^{-1} a_{\overline{2}}-\left(D_{\overline{0}+}^{-1} a_{\overline{3}}\right)^{3}\right) \frac{\delta F}{\delta J_{\overline{0}+}}, \\
\eta_{\overline{2}}= & \frac{\delta F}{\delta J_{\overline{2}+}}-D_{\overline{0}+}^{-1} a_{\overline{3}} D_{\overline{0}+}^{-1} a_{\overline{2}} \frac{\delta F}{\delta J_{\overline{3}+}}+\left(D_{\overline{0}+}^{-1} a_{\overline{2}}-\left(D_{\overline{0}+}^{-1} a_{\overline{3}}\right)^{2}\right) \frac{\delta F}{\delta J_{\overline{0}+}}, \\
\eta_{\overline{3}}= & \left(D_{\overline{0}+}^{-1} a_{\overline{2}}-a_{\overline{2}}^{-1} D_{\overline{0}+}\right) \frac{\delta F}{\delta J_{\overline{3}+}}-a_{\overline{2}}^{-1} a_{\overline{3}} \frac{\delta F}{\delta J_{\overline{2}+}}+D_{\overline{0}+}^{-1} a_{\overline{3}} \frac{\delta F}{\delta J_{\overline{0}+}},
\end{aligned}
$$

where we have denoted

$$
a_{\overline{2}}=\operatorname{ad}_{J_{\overline{2}+}}, \quad a_{\overline{3}}=\operatorname{ad}_{J_{\overline{3}+}}
$$


This gives the Poisson bracket of $F$ with the currents:

$$
\begin{aligned}
\left\{F, J_{\overline{2}+}\right\}= & \left(D_{\overline{0}+}-a_{\overline{3}} a_{\overline{2}}^{-1} a_{\overline{3}}\right) \frac{\delta F}{\delta J_{\overline{2}+}}-a_{\overline{3}} a_{\overline{2}}^{-1} D_{\overline{0}+} \frac{\delta F}{\delta J_{\overline{3}+}}+a_{\overline{2}} \frac{\delta F}{\delta J_{\overline{0}+}}, \\
\left\{F, J_{\overline{3}+}\right\}= & \left(a_{\overline{3}}-\left(a_{\overline{2}} D_{\overline{0}+}^{-1}\right)^{2} a_{\overline{3}}-a_{\overline{2}} D_{\overline{0}+}^{-1} a_{\overline{3}} D_{\overline{0}+}^{-1} a_{\overline{2}}\right. \\
& \left.+a_{\overline{2}}\left(D_{\overline{0}+}^{-1} a_{\overline{3}}\right)^{3}\right) \frac{\delta F}{\delta J_{\overline{0}+}}-D_{\overline{0}+} a_{\overline{2}}^{-1} a_{\overline{3}} \frac{\delta F}{\delta J_{\overline{2}+}} \\
& +\left(-a_{\overline{2}}\left(a_{\overline{2}}^{-1} D_{\overline{0}+}-D_{\overline{0}+}^{-1} a_{\overline{2}}\right)^{2}+a_{\overline{2}}\left(D_{\overline{0}+}^{-1} a_{\overline{3}}\right)^{2} D_{\overline{0}+}^{-1} a_{\overline{2}}\right) \frac{\delta F}{\delta J_{\overline{3}+}}, \\
\left\{F, J_{\overline{0}+}\right\}= & \left(-a_{\overline{3}} D_{\overline{0}+}^{-1} a_{\overline{2}} D_{\overline{0}+}^{-1} a_{\overline{3}}+a_{\overline{3}}\left(D_{\overline{0}+}^{-1} a_{\overline{3}}\right)^{3}+a_{\overline{2}} D_{\overline{0}+}^{-1} a_{\overline{2}}\right. \\
& \left.-a_{\overline{2}}\left(D_{\overline{0}+}^{-1} a_{\overline{3}}\right)^{2}-\left(a_{\overline{3}} D_{\overline{0}+}^{-1}\right)^{2} a_{\overline{2}}\right) \frac{\delta F}{\delta J_{\overline{0}+}}+a_{\overline{2}} \frac{\delta F}{\delta J_{\overline{2}+}} \\
& +\left(a_{\overline{3}}-a_{\overline{2}} D_{\overline{0}+}^{-1} a_{\overline{3}} D_{\overline{0}+}^{-1} a_{\overline{2}}+\left(a_{\overline{3}} D_{\overline{0}+}^{-1}\right)^{3} a_{\overline{2}}-a_{\overline{3}}\left(D_{\overline{0}+}^{-1} a_{\overline{2}}\right)^{2}\right) \frac{\delta F}{\delta J_{\overline{3}+}} .
\end{aligned}
$$

Therefore the Poisson bracket of the currents is

$$
\begin{aligned}
\left\{J_{\overline{2}+}, J_{\overline{2}+}\right\}= & -D_{\overline{0}+}+a_{\overline{3}} a_{\overline{2}}^{-1} a_{\overline{3}}, \\
\left\{J_{\overline{2}+}, J_{\overline{3}+}\right\}= & a_{\overline{3}} a_{\overline{2}}^{-1} D_{\overline{0}+}, \\
\left\{J_{\overline{2}+}, J_{\overline{0}+}\right\}= & -a_{\overline{2}}, \\
\left\{J_{\overline{3}+}, J_{\overline{0}+}\right\}= & -a_{\overline{3}}+a_{\overline{2}} D_{\overline{0}+}^{-1} a_{\overline{3}} D_{\overline{0}+}^{-1} a_{\overline{2}}-a_{\overline{2}} D_{\overline{0}+}^{-1} a_{\overline{3}} D_{\overline{0}+}^{-1} a_{\overline{3}} D_{\overline{0}+}^{-1} a_{\overline{3}} \\
& +a_{\overline{2}} D_{\overline{0}+}^{-1} a_{\overline{2}} D_{\overline{0}+}^{-1} a_{\overline{3}}, \\
\left\{J_{\overline{3}+}, J_{\overline{3}+}\right\}= & \left(D_{\overline{0}+}-a_{\overline{2}} D_{\overline{0}+}^{-1} a_{\overline{2}}\right) a_{\overline{2}}^{-1}\left(D_{\overline{0}+}-a_{\overline{2}} D_{\overline{0}+}^{-1} a_{\overline{2}}\right) \\
& -a_{\overline{2}} D_{\overline{0}+}^{-1} a_{\overline{3}} D_{\overline{0}+}^{-1} a_{\overline{3}} D_{\overline{0}+}^{-1} a_{\overline{2}}, \\
\left\{J_{\overline{0}+}, J_{\overline{0}+}\right\}= & -a_{\overline{2}} D_{\overline{0}+}^{-1} a_{\overline{2}}+a_{\overline{3}} D_{\overline{0}+}^{-1} a_{\overline{3}} D_{\overline{0}+}^{-1} a_{\overline{2}}+a_{\overline{2}} D_{\overline{0}+}^{-1} a_{\overline{3}} D_{\overline{0}+}^{-1} a_{\overline{3}} \\
& +a_{\overline{3}} D_{\overline{0}+}^{-1} a_{\overline{2}} D_{\overline{0}+}^{-1} a_{\overline{3}}-a_{\overline{3}} D_{\overline{0}+}^{-1} a_{\overline{3}} D_{\overline{0}+}^{-1} a_{\overline{3}} D_{\overline{0}+}^{-1} a_{\overline{3}} .
\end{aligned}
$$

We should remember that these Poisson brackets are defined only on those functions which are gauge invariant. Note that $a_{\overline{2}}^{-1}$ exists because of (5.4). 


\subsection{Action of the boosts on the canonical Poisson bracket}

The canonical Poisson brackets are not invariant under the boosts. But it turns out that the boosts act on the canonical bracket in some relatively simple way. In fact, the canonical bracket is the sum of three terms each having a definite scaling dimension:

$$
\theta_{\text {str }}=\theta^{[2]}+\theta^{[0]}+\theta^{[-2]} \text {. }
$$

We have the following expressions for $\theta^{[2]}$ and $\theta^{[0]}$. The lowest grade $\theta^{[2]}$ is

$$
\begin{aligned}
& \left\{J_{\overline{2}+}, J_{\overline{2}+}\right\}^{[2]}=-D_{\overline{0}+}+a_{\overline{3}} a_{\overline{2}}^{-1} a_{\overline{3}}, \\
& \left\{J_{\overline{2}+}, J_{\overline{3}+}\right\}^{[2]}=a_{\overline{3}} a_{\overline{2}}^{-1} D_{0_{+}}, \\
& \left\{J_{\overline{3}+}, J_{\overline{3}+}\right\}^{[2]}=D_{\overline{0}+} a_{\overline{2}}^{-1} D_{\overline{0}_{+} \cdot}
\end{aligned}
$$

The next grade is $\theta^{[0]}$ :

$$
\begin{aligned}
& \left\{J_{\overline{2}+}, J_{\overline{0}_{+}}\right\}^{[0]}=-a_{\overline{2}}, \\
& \left\{J_{\overline{3}+}, J_{\overline{0}_{+}}\right\}^{[0]}=-a_{\overline{3}}, \\
& \left\{J_{\overline{3}+}, J_{\overline{3}_{+}}\right\}^{[0]}=-2 a_{\overline{2}} .
\end{aligned}
$$

Moreover, the highest grade is $\theta^{[-2]}$ :

$$
\begin{aligned}
\left\{J_{\overline{3}+}, J_{\overline{0}+}\right\}^{[-2]}= & a_{\overline{2}} D_{\overline{0}+}^{-1} a_{\overline{3}} D_{\overline{0}+}^{-1} a_{\overline{2}}-a_{\overline{2}} D_{\overline{0}+}^{-1} a_{\overline{3}} D_{\overline{0}+}^{-1} a_{\overline{3}} D_{\overline{0}+}^{-1} a_{\overline{3}} \\
& +a_{\overline{2}} D_{\overline{0}+}^{-1} a_{\overline{2}} D_{\overline{0}+}^{-1} a_{\overline{3}}, \\
\left\{J_{\overline{3}+}, J_{\overline{3}+}\right\}^{[-2]}= & a_{\overline{2}} D_{\overline{0}}^{-1} a_{\overline{2}} D_{\overline{0}}^{-1} a_{\overline{2}}-a_{\overline{2}} D_{\overline{0}+}^{-1} a_{\overline{3}} D_{\overline{0}+}^{-1} a_{\overline{3}} D_{\overline{0}+}^{-1} a_{\overline{2}}, \\
\left\{J_{\overline{0}+}, J_{\overline{0}+}\right\}^{[-2]}= & -a_{\overline{2}} D_{\overline{0}+}^{-1} a_{\overline{2}}+a_{\overline{3}} D_{\overline{0}+}^{-1} a_{\overline{3}} D_{\overline{0}+}^{-1} a_{\overline{2}}+a_{\overline{2}} D_{\overline{0}+}^{-1} a_{\overline{3}} D_{\overline{0}+}^{-1} a_{\overline{3}} \\
& +a_{\overline{3}} D_{\overline{0}+}^{-1} a_{\overline{2}} D_{\overline{0}+}^{-1} a_{\overline{3}}-a_{\overline{3}} D_{\overline{0}+}^{-1} a_{\overline{3}} D_{\overline{0}+}^{-1} a_{\overline{3}} D_{\overline{0}+}^{-1} a_{\overline{3}} .
\end{aligned}
$$

\subsection{Different ways of presenting the Poisson bivector}

In our formalism the Poisson bivector is defined modulo some equivalence relation. This is because we calculate $\{F(J), G(J)\}$ assuming that $F$ and $G$ 
are invariant under the gauge transformations

$$
\delta_{\xi_{\overline{0}}} J=-D \xi_{\overline{0}}
$$

This means that when we are calculating $\{F, G\}$, we are assuming that $F$ and $G$ are such that

$$
D_{\overline{0}+} \frac{\delta F}{\delta J_{\overline{0}+}}+\left[J_{\overline{2}+}, \frac{\delta F}{\delta J_{\overline{2}+}}\right]+\left[J_{\overline{3}+}, \frac{\delta F}{\delta J_{\overline{3}+}}\right]=0
$$

and the same is true about $G$. Therefore the Poisson bivector $\theta^{i j}$ is defined only up to the equivalence

$$
\theta \simeq \theta+v \wedge \delta_{\xi_{\overline{0}}}, \quad \xi_{\overline{0}} \in \mathbf{g}_{\overline{0}},
$$

where $v$ is a vector field on the phase space. Therefore the Poisson bivector (5.9) can be replaced by

$$
\begin{aligned}
& \left\{J_{\overline{0}+}, J_{\overline{0}+}\right\}^{[0]}=2 D_{\overline{0}_{+}}, \\
& \left\{J_{\overline{3}+}, J_{\overline{3}+}\right\}^{[0]}=-2 a_{\overline{2}} .
\end{aligned}
$$

(The Poisson brackets of the components not listed are zero.)

\subsection{Symplectic leaves}

When we write the Poisson bracket $\{,\}^{[0]}$ in the form (5.13) and (5.14), it becomes obvious that this Poisson structure is degenerate. Indeed, the brackets do not involve $J_{\overline{2}}$ at all. Degenerate Poisson brackets define submanifolds in the phase space known as "symplectic leaves". Consider a point $x$ of the phase space. Given a 1 -form $\lambda$ at $x$, we can contract it with the Poisson bivector $\theta(x)$ and get a vector $\theta^{i j}(x) \lambda_{j}$ in the tangent space at the point $x$. Therefore $\theta(x)$ defines a linear map $\theta(x): T_{x}^{*} M \rightarrow T_{x} M$. The image of this map is a subspace $\operatorname{Im} \theta(x) \subset T_{x} M$. It turns out that when we vary $x$, the collection of spaces $\operatorname{Im} \theta(x)$ is an integrable distribution, in the sense that there exists a foliation of $M$ by submanifolds $N \subset M$ such that the tangent space to $N$ at every point coincides with $\operatorname{Im} \theta$. (There is a family of submanifolds $N$, parametrized by $\operatorname{codim} N$ parameters; they are known as "the integral manifolds of the distribution $\operatorname{Im} \theta$ ".) The submanifolds $N$ 
are called "symplectic leaves":

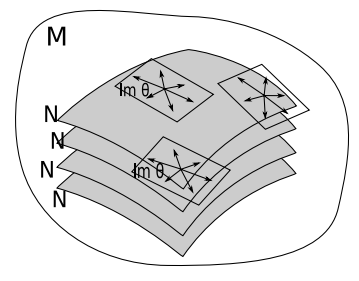

The symplectic leaves can also be described in terms of the Casimir functionals. A function $C$ on the phase space is called Casimir function (or functional), if its Poisson bracket with any other functional vanishes. The non-degenerate Poisson brackets do not have any Casimir functionals, but the degenerate brackets do. The symplectic leaves can be characterized as the common level sets of all the Casimir functionals.

Let us first discuss $\{,\}^{[0]}$ in the bosonic sector, equation (5.13). Bosonic degrees of freedom are $J_{\overline{0}+}$ and $J_{\overline{2}+}$. The symplectic leaves are the manifolds with the constant eigenvalues of $J_{\overline{2}+}$. Indeed, the only Casimir functionals are the gauge invariant functionals of $J_{\overline{2}+}$ only. But the gauge invariance (5.11) acts on $J_{\overline{2}+}$ by the conjugation, and therefore the Casimir functions are precisely the functionals of the eigenvalues of $J_{\overline{2}+}$. Therefore the symplectic leaves are the level sets of the eigenvalues (spectral invariants) of $J_{\overline{2}+}$.

The symplectic leaves are compatible with the Virasoro constraint (4.19) in a sense that, if one point on the symplectic leaf satisfies (4.19), then all the points on this leaf also satisfy (4.19). The bosonic part $\mathbf{g}_{e v}=\mathbf{g}_{\overline{0}} \oplus \mathbf{g}_{\overline{2}}$ of $p s u(2,2 \mid 4)$ is $s o(2,4) \oplus s o(6)$. It turns out, because of the properties of $\mathbf{g}=\operatorname{psu}(2,2 \mid 4)$, that any $J_{\overline{2}}+$ satisfying (4.19) is of the form

$$
J_{\overline{2}+}=\left(\begin{array}{cccccc}
0 & \alpha & 0 & 0 & 0 & 0 \\
-\alpha & 0 & 0 & 0 & 0 & 0 \\
0 & 0 & 0 & 0 & 0 & 0 \\
0 & 0 & 0 & 0 & 0 & 0 \\
0 & 0 & 0 & 0 & 0 & 0 \\
0 & 0 & 0 & 0 & 0 & 0
\end{array}\right)_{s o(2,4)} \bigoplus\left(\begin{array}{cccccc}
0 & \alpha & 0 & 0 & 0 & 0 \\
-\alpha & 0 & 0 & 0 & 0 & 0 \\
0 & 0 & 0 & 0 & 0 & 0 \\
0 & 0 & 0 & 0 & 0 & 0 \\
0 & 0 & 0 & 0 & 0 & 0 \\
0 & 0 & 0 & 0 & 0 & 0
\end{array}\right)_{s o(6)}
$$

up to the conjugation. Here $\alpha=\alpha\left(\tau^{+}, \tau^{-}\right)$are some functions of $\tau^{+}$and $\tau^{-}$. The string worldsheet fields are defined up to conformal transformations $\left(\tau^{+}, \tau^{-}\right) \mapsto\left(F^{+}\left(\tau^{+}\right), F^{-}\left(\tau^{-}\right)\right)$where $F^{+}$and $F^{-}$are arbitrary functions (with $\left(F^{+}\right)^{\prime} \neq 0,\left(F^{-}\right)^{\prime} \neq 0$ ). Equation (4.14) implies that $\partial_{-} \alpha=0$. This means that we can do such a conformal transformation $\tau^{+} \mapsto F^{+}\left(\tau^{+}\right)$, or in other words choose the worldsheet coordinates $\left(\tau^{+}, \tau^{-}\right)$in such a way that 
$\alpha$ is constant and equal to 1 :

$$
\alpha\left(\tau^{+}, \tau^{-}\right) \equiv 1
$$

In the following discussion we will use these particular coordinates. Note that equation (5.15) defines a symplectic leaf. The hidden relativistic symmetry of Section 5.1 preserves the condition (5.15).

\subsection{Relation between $\theta^{[0]}$ and the chiral WZW bracket}

Now we want to explain that $\theta^{[0]}$ can be thought of as a Hamiltonian reduction of the Kirillov bracket on the coadjoint orbit of the Kac-Moody algebra. The discussion in this subsection is similar to [11]; equation (5.23) easily follows from the results of [36].

Let us first discuss the Poisson bracket of the bosonic fields $J_{\overline{0}+}$, and then turn to the fermions. Let us choose the gauge where $J_{\overline{2}}$ is equal to

$$
J_{\overline{2}+}=\left(\begin{array}{cccccc}
0 & 1 & 0 & 0 & 0 & 0 \\
-1 & 0 & 0 & 0 & 0 & 0 \\
0 & 0 & 0 & 0 & 0 & 0 \\
0 & 0 & 0 & 0 & 0 & 0 \\
0 & 0 & 0 & 0 & 0 & 0 \\
0 & 0 & 0 & 0 & 0 & 0
\end{array}\right)_{s o(2,4)} \bigoplus\left(\begin{array}{cccccc}
0 & 1 & 0 & 0 & 0 & 0 \\
-1 & 0 & 0 & 0 & 0 & 0 \\
0 & 0 & 0 & 0 & 0 & 0 \\
0 & 0 & 0 & 0 & 0 & 0 \\
0 & 0 & 0 & 0 & 0 & 0 \\
0 & 0 & 0 & 0 & 0 & 0
\end{array}\right)_{s o(6)}
$$

(Remember that the bosonic part of $p s u(2,2 \mid 4)$ is $\mathbf{g}_{\overline{0}} \oplus \mathbf{g}_{\overline{2}}=s o(2,4) \oplus$ so(6).) In this gauge $F$ and $G$ become functions of $J_{\overline{0}+}$, invariant under the residual gauge transformations:

$$
\delta_{\zeta} J_{\overline{0}+}=D_{\overline{0}+} \zeta, \quad \zeta \in \mathbf{h},
$$

where $\zeta$ belongs to the subalgebra $\mathbf{h} \subset \mathbf{g}_{\overline{0}}$ which stabilizes $J_{\overline{2}+}$ :

$$
\mathbf{h}:\left(\begin{array}{llllll}
0 & 0 & 0 & 0 & 0 & 0 \\
0 & 0 & 0 & 0 & 0 & 0 \\
0 & 0 & * & * & * & * \\
0 & 0 & * & * & * & * \\
0 & 0 & * & * & * & * \\
0 & 0 & * & * & * & *
\end{array}\right)_{s o(2,4)} \bigoplus\left(\begin{array}{llllll}
0 & 0 & 0 & 0 & 0 & 0 \\
0 & 0 & 0 & 0 & 0 & 0 \\
0 & 0 & * & * & * & * \\
0 & 0 & * & * & * & * \\
0 & 0 & * & * & * & * \\
0 & 0 & * & * & * & *
\end{array}\right)_{s o(6)} .
$$

We want to describe the Poisson bracket (5.13) on the functions invariant under the gauge transformations (5.17). We can look at it in the following 
way. Consider first the bracket (5.13) on arbitrary functions $F\left(J_{\overline{0}+}\right)$, not necessarily gauge invariant. This is essentially the chiral WZW bracket [37], or equivalently the Kirillov bracket on the coadjoint orbit of the Kac-Moody algebra. Let us parametrize the currents $J_{\overline{0}+}$ in terms of the monodromy $f$ :

$$
J_{\overline{0}+}=-\partial_{+} f f^{-1} .
$$

The symplectic structure corresponding to (5.13) is

$$
\Omega_{W Z W}=\int d \tau^{+} \operatorname{tr}\left(\delta f f^{-1} \delta\left(\partial_{+} f f^{-1}\right)\right) .
$$

Now we want to describe the bracket (5.13) specifically on the functions invariant under the gauge transformations (5.17). Instead of talking about the gauge invariant functions we can consider functions on a submanifold in the phase space (the "gauge slice"), which is a symplectic complement of the orbits of (5.17), with respect to the symplectic form (5.18). "Symplectic complement" means that for any vector $\xi$ tangent to the gauge slice and any $\zeta \in \mathbf{h}$ we should have

$$
\Omega_{W Z W}\left(\delta_{\xi}, \delta_{\zeta}\right)=0 .
$$

We choose the following gauge slice:

$$
J_{\overline{0}+}=\left(\begin{array}{ccccc}
0 & q_{+}^{0} & q_{+}^{1} & q_{+}^{2} & q_{+}^{3} \\
\tilde{q}_{+}^{0} & 0 & 0 & 0 & 0 \\
-\tilde{q}_{+}^{1} & 0 & 0 & 0 & 0 \\
-\tilde{q}_{+}^{2} & 0 & 0 & 0 & 0 \\
-\tilde{q}_{+}^{3} & 0 & 0 & 0 & 0
\end{array}\right)_{s o(1,4)} \bigoplus\left(\begin{array}{ccccc}
0 & q_{+}^{1} & q_{+}^{2} & q_{+}^{3} & q_{+}^{4} \\
-q_{+}^{1} & 0 & 0 & 0 & 0 \\
-q_{+}^{2} & 0 & 0 & 0 & 0 \\
-q_{+}^{3} & 0 & 0 & 0 & 0 \\
-q_{+}^{4} & 0 & 0 & 0 & 0
\end{array}\right)_{s o(5)} .
$$

This gauge slice satisfies equation (5.19). This implies that the symplectic structure in terms of the variables $q_{+}^{i}$ is given by the restriction of the symplectic form (5.18) to the subspace of the phase space specified by the constraint that $\partial_{+} f f^{-1}=-J_{\overline{0}+}$ is of the form (5.20).

The gauge choice (5.20) was used in [8-15]. Geometrically it corresponds to the so-called "normal frame". The normal frame is the basis in the normal bundle to the curve such that the covariant derivative of any element of this basis along the curve is parallel to the tangent vector to the curve [38].

Now let us consider the fermionic part. The Poisson bracket

$$
\left\{J_{\overline{3}+}, J_{\overline{3}+}\right\}=a_{\overline{2}}
$$


is degenerate. It has symplectic leaves which are described by the equation

$$
J_{\overline{3}}-J_{\overline{3}}^{(0)}=\left[J_{\overline{2}+}, K_{\overline{1}}\right]
$$

where $K_{\overline{1}}$ runs over $\mathbf{g}_{\overline{1}}$. The gauge (4.26) corresponds to the symplectic leaf with $J_{\overline{3}}^{(0)}=0$, in other words

$$
J_{\overline{3}} \in \operatorname{Im}\left(\operatorname{add}_{\overline{\overline{2}}_{\overline{+}}}: \mathbf{g}_{\overline{1}} \rightarrow \mathbf{g}_{\overline{3}}\right) .
$$

On this symplectic leaf we have $J_{\overline{3}+}=\left[J_{\overline{2}+}, K_{\overline{1}}\right]$ and the symplectic form is:

$$
\int d \tau^{+}\left(\operatorname{tr}\left(\delta f f^{-1} \delta\left(\partial_{+} f f^{-1}\right)\right)+\operatorname{tr}\left(\delta K_{\overline{1}}\left[J_{\overline{2}+}, \delta K_{\overline{1}}\right]\right)\right) .
$$

This is obviously a closed form, ${ }^{3}$ and therefore $\{,\}^{[0]}$ satisfies the Jacobi identity. The bosonic part of the symplectic form (5.23) follows from the action of the generalized sine-Gordon model which was suggested in [36]. The possible relation with the WZW model on the quantum level was discussed in [39].

\subsection{Relation to the results of $[16]$}

If we apply this formailsm to $\mathbf{g}=s o(3)$ we get $\mathbf{g}_{\overline{0}}=s o(2)$ and $\mathbf{g}_{\overline{2}}$ is the vector representation of $s o(2)$. We can write:

$$
J_{\overline{2}+}=\left[\begin{array}{c}
r \\
\cos \varphi \\
r \sin \varphi
\end{array}\right]
$$

The Poisson bracket $\theta^{[2]}$ at $r=1$ becomes

$$
\{F, G\}^{[2]}=\int d \tau^{+}\left(\frac{\delta F}{\delta \varphi} \partial_{+} \frac{\delta G}{\delta \varphi}+\frac{\delta F}{\delta r} \partial_{+} \frac{\delta G}{\delta r}-\partial_{+} \varphi\left(\frac{\delta F}{\delta r} \frac{\delta G}{\delta \varphi}-\frac{\delta F}{\delta \varphi} \frac{\delta G}{\delta r}\right)\right) .
$$

This means that if $F$ depends only on $\varphi$ we get $\left\{r\left(\tau_{1}^{+}\right), F\right\}^{[2]}=\frac{\delta F}{\delta \varphi\left(\tau_{1}^{+}\right)} \partial_{+}$ $\varphi\left(\tau_{1}^{+}\right)$. If we consider $F$ as a function of $q_{+}=\partial_{+} \varphi$, then $\frac{\delta F}{\delta \varphi}=-\partial_{+} \frac{\delta F}{\delta q_{+}}$. If

\footnotetext{
${ }^{3}$ We have to remember that $J_{\overline{2}+}$ is gauge fixed to be equal to (5.16).
} 
we fix the Virasoro constraint $r=1$, the Dirac bracket becomes

$$
\begin{aligned}
\{F, G\}_{D} & =\{F, G\}-\{F, r\}\{r, r\}^{-1}\{r, G\} \\
& =\{F, G\}-\int d \tau^{+} \frac{\delta F}{\delta q_{+}} \partial_{+} q_{+} \partial_{+}^{-1} q_{+} \partial_{+} \frac{\delta G}{\delta q_{+}} .
\end{aligned}
$$

This agrees with $\theta_{1}$ of [16]. We see that resolving the Virasoro constraint gives an additional nonlocal piece in the Dirac bracket [40].

\subsection{The relation between $\theta^{[2]}, \theta^{[0]}$ and $\theta^{[-2]}$}

We have seen that the boost-invariant Poisson bracket (5.9) can be written in the equivalent form (5.13) and (5.14). Alternatively, it can be also (using the same equivalence relations, described in Section 5.4) written in the following form:

$$
\begin{aligned}
& \left\{J_{\overline{2}+}, J_{\overline{2}+}\right\}^{[0]}=2 a_{\overline{2}} D_{\overline{0}+}^{-1} a_{\overline{2}}, \\
& \left\{J_{\overline{2}+}, J_{\overline{3}+}\right\}^{[0]}=2 a_{\overline{2}} D_{\overline{0}+}^{-1} a_{\overline{3}}, \\
& \left\{J_{\overline{3}+}, J_{\overline{3}+}\right\}^{[0]}=-2 a_{\overline{2}}+2 a_{\overline{3}} D_{\overline{0}+}^{-1} a_{\overline{3}} .
\end{aligned}
$$

The brackets of the components not listed are zero. In particular, $J_{0+}$ commutes with everything. This means that equation (5.26) gives the boostinvariant Poisson bracket in the gauge $J_{0+}=0$. More explicitly:

$$
\{F, G\}^{[0]}=2 \int d \tau^{+}\left[\frac{\delta F}{\delta J_{\overline{2}+}}, \frac{\delta F}{\delta J_{\overline{3}+}}\right]\left[\begin{array}{cc}
a_{\overline{2}} D_{\overline{0}+}^{-1} a_{\overline{2}} & a_{\overline{2}} D_{\overline{0}+}^{-1} a_{\overline{3}} \\
a_{\overline{3}} D_{\overline{0}+}^{-1} a_{\overline{2}} & -a_{\overline{2}}+a_{\overline{3}} D_{\overline{0}+}^{-1} a_{\overline{3}}
\end{array}\right]\left[\begin{array}{c}
\delta G \\
\delta J_{\overline{2}+} \\
\frac{\delta G}{\delta J_{\overline{3}+}}
\end{array}\right] .
$$

In other words, $\theta^{[0]}$ in this "picture" is

$$
\theta^{[0]}=\left[\begin{array}{cc}
2 a_{\overline{2}} D_{\overline{0}+}^{-1} a_{\overline{2}} & 2 a_{\overline{2}} D_{\overline{0}+}^{-1} a_{\overline{3}} \\
2 a_{\overline{3}} D_{\overline{0}+}^{-1} a_{\overline{2}} & -2 a_{\overline{2}}+2 a_{\overline{3}} D_{\overline{0}+}^{-1} a_{\overline{3}}
\end{array}\right] .
$$

Let us also explicitly write $\theta^{[2]}$ in the same gauge:

$$
\{F, G\}^{[2]}=\int d \tau^{+}\left[\frac{\delta F}{\delta J_{\overline{2}+}, \frac{\delta F}{\delta J_{\overline{3}+}}}\right]\left[\begin{array}{cc}
-D_{\overline{0}+}+a_{\overline{3}} a_{\overline{2}}^{-1} a_{\overline{3}} & a_{\overline{3}} a_{\overline{2}}^{-1} D_{\overline{0}+} \\
D_{\overline{0}+} a_{\overline{2}}^{-1} a_{\overline{3}} & D_{\overline{0}+} a_{\overline{2}}^{-1} D_{\overline{0}+}
\end{array}\right]\left[\begin{array}{c}
\delta G \\
\delta J_{\overline{2}+} \\
\delta G \\
\frac{\delta J_{\overline{3}+}}{}
\end{array}\right] .
$$


The corresponding symplectic structure $\Omega^{[2]}=\left(\theta^{[2]}\right)^{-1}$ is

$$
\begin{aligned}
\Omega^{[2]} & =\left(\theta^{[2]}\right)^{-1} \\
& =\int d \tau^{+}\left[\delta J_{\overline{2}+}, \delta J_{\overline{3}+}\right] D_{\overline{0}+}^{-1}\left[\begin{array}{cc}
-D_{\overline{0}+} & a_{\overline{3}} \\
a_{\overline{3}} & a_{\overline{2}}-a_{\overline{3}} D_{\overline{0}+}^{-1} a_{\overline{3}}
\end{array}\right] D_{\overline{0}+}^{-1}\left[\begin{array}{l}
\delta J_{\overline{2}+} \\
\delta J_{\overline{3}+}
\end{array}\right] .
\end{aligned}
$$

Now let us bring $\theta^{[-2]}$ to the same gauge. Section 5.4 allows us to present $\{,\}^{[-2]}$ as follows:

$$
\{F, G\}^{[-2]}=\int d \tau^{+}\left[\frac{\delta F}{\delta J_{\overline{2}+}}, \frac{\delta F}{\delta J_{\overline{3}+}}\right]\left[\begin{array}{cc}
\theta_{\overline{2} \overline{2}}^{[-2]} & \theta_{\overline{2} \overline{3}}^{[-2]} \\
\theta_{\overline{3} \overline{2}}^{[-2]} & \theta_{\overline{3} \overline{3}}^{[-2]}
\end{array}\right]\left[\begin{array}{l}
\delta G / \delta J_{\overline{2}+} \\
\delta G / \delta J_{\overline{3}+}
\end{array}\right],
$$

where

$$
\begin{aligned}
\theta_{\overline{2} \overline{2}}^{[-2]}= & -\mathcal{O}_{2222}+\mathcal{O}_{23322}+\mathcal{O}_{22332}+\mathcal{O}_{23232}-\mathcal{O}_{233332} \\
\theta_{\overline{2} \overline{3}}^{[-2]}= & -\mathcal{O}_{2223}-\mathcal{O}_{2232}-\mathcal{O}_{2322} \\
& +\mathcal{O}_{23332}+\mathcal{O}_{23323}+\mathcal{O}_{23233}+\mathcal{O}_{22333}-\mathcal{O}_{233333} \\
\theta_{\overline{3} \overline{3}}^{[-2]}= & \mathcal{O}_{222}-\mathcal{O}_{2233}-\mathcal{O}_{2323}-\mathcal{O}_{3232}-\mathcal{O}_{3322}-\mathcal{O}_{2332}-\mathcal{O}_{3223} \\
& +\mathcal{O}_{23333}+\mathcal{O}_{32333}+\mathcal{O}_{33233}+\mathcal{O}_{33323}+\mathcal{O}_{33332}-\mathcal{O}_{333333}
\end{aligned}
$$

Here we introduced the notations $\mathcal{O}_{\bar{j}_{1} \bar{j}_{2} \ldots \bar{j}_{n}}$ :

$$
\mathcal{O}_{\bar{j}_{1} \bar{j}_{2} \bar{j}_{3} \ldots \bar{j}_{n}}=a_{\bar{j}_{1}} D_{\overline{0}+}^{-1} a_{\bar{j}_{2}} D_{\overline{0}+}^{-1} a_{\bar{j}_{3}} D_{\overline{0}+}^{-1} \cdots D_{\overline{0}+}^{-1} a_{\bar{j}_{n}} .
$$

The indices of $\mathcal{O}$ run over $\bar{j} \in\{\overline{2}, \overline{3}\}$. An explicit computation using (5.28) and (5.27) and (5.29) shows that

$$
4 \theta^{[-2]}=\theta^{[0]}\left(\theta^{[2]}\right)^{-1} \theta^{[0]} .
$$

We see that the Poisson bivector has the same structure as in the case of the string in $\mathbf{R} \times S^{2}$ considered in [16]:

$$
\theta^{\text {can }}=\theta^{[2]}+\theta^{[0]}+\frac{1}{4} \theta^{[0]}\left(\theta^{[2]}\right)^{-1} \theta^{[0]} .
$$




\subsection{Poisson bracket and the monodromy matrix}

The monodromy matrix can be defined as a path ordered exponential:

$$
M(z)^{a b}=\left[P \exp \int_{-\infty}^{\infty} d \tau^{+}\left(-z J_{1+}-J_{0+}-\frac{1}{z} J_{3+}-\frac{1}{z^{2}} J_{2+}\right)\right]^{a b} .
$$

Although we are using the gauge $J_{1+}=0$ we have included $J_{1+}$ in the definition of $M$ for the convenience of notations; we will consider the variational derivative $\frac{\delta M}{\delta J_{1+}}$ at $J_{1+}=0$. The monodromy matrix (5.34) is a functional of the currents, and we can calculate its Poisson brackets with the other functionals. Let us first study the properties of the variational derivatives $\frac{\delta M}{\delta J_{+}\left(\tau^{+}\right)}$. We will define $\frac{\delta M}{\delta J_{+}\left(\tau^{+}\right)}$as a matrix such that

$$
\int_{-\infty}^{+\infty} d \tau^{+} \operatorname{str} \delta J_{+} \frac{\delta M^{a b}}{\delta J_{+}\left(\tau^{+}\right)}=\delta M^{a b}
$$

Consider the following identities:

$$
\begin{aligned}
& D_{0+} \frac{\delta M^{a b}}{\delta J_{3+}}+\left[J_{3+}, \frac{\delta M^{a b}}{\delta J_{2+}}\right]+\frac{1}{z^{4}}\left[J_{2+}, \frac{\delta M^{a b}}{\delta J_{1+}}\right]=0, \\
& D_{0+} \frac{\delta M^{a b}}{\delta J_{2+}}+\frac{1}{z^{4}}\left[J_{3+}, \frac{\delta M^{a b}}{\delta J_{1+}}\right]+\frac{1}{z^{4}}\left[J_{2+}, \frac{\delta M^{a b}}{\delta J_{0+}}\right]=0, \\
& D_{0+} \frac{\delta M^{a b}}{\delta J_{1+}}+\left[J_{2+}, \frac{\delta M^{a b}}{\delta J_{3+}}\right]+\left[J_{3+}, \frac{\delta M^{a b}}{\delta J_{0+}}\right]=0, \\
& D_{0+} \frac{\delta M^{a b}}{\delta J_{0+}}+\left[J_{2+}, \frac{\delta M^{a b}}{\delta J_{2+}}\right]+\left[J_{3+}, \frac{\delta M^{a b}}{\delta J_{3+}}\right]=0,
\end{aligned}
$$

which follow from the invariance of (5.34) under the gauge transformations with $z$-dependent parameters; for example (5.36) follows from the formula:

$$
\delta_{\alpha_{3}}\left(D_{0+}+z J_{1+}+\frac{1}{z} J_{3+}+\frac{1}{z^{2}} J_{2+}\right)=\left[D_{0+}+z J_{1+}+\frac{1}{z} J_{3+}+\frac{1}{z^{2}} J_{2+}, \frac{1}{z} \alpha_{3}\right],
$$

which should be understood as $\delta_{\alpha_{3}} J_{3+}=D_{0+} \alpha_{3}$ and $\delta_{\alpha_{3}} J_{1+}=\frac{1}{z^{4}}\left[J_{2+}, \alpha_{3}\right]$. Note that (5.39) is the statement of $\mathbf{g}_{\overline{0}}$ gauge invariance. Equations (5.36), 
(5.37) and (5.8) imply:

$$
\begin{aligned}
& \left\{J_{2+}, M^{a b}\right\}^{[2]}=\frac{1}{z^{4}}\left[J_{2+}, \frac{\delta M^{a b}}{\delta J_{0+}}\right], \\
& \left\{J_{3+}, M^{a b}\right\}^{[2]}=-\frac{1}{z^{4}} D_{0+} \frac{\delta M^{a b}}{\delta J_{1+}} .
\end{aligned}
$$

On the other hand, equation (5.26) imply

$$
\begin{aligned}
& \left\{J_{2+}, M^{a b}\right\}^{[0]}=-2\left[J_{2+}, \frac{\delta M^{a b}}{\delta J_{0+}}\right], \\
& \left\{J_{3+}, M^{a b}\right\}^{[0]}=2 D_{0+} \frac{\delta M^{a b}}{\delta J_{1+}} .
\end{aligned}
$$

These equations together with (5.37) imply that

$$
\begin{aligned}
& M(z)^{a b} \text { is a Casimir functional of the Poisson bracket } \\
& \{,\}^{[0]}+2 z^{4}\{,\}^{[2]} .
\end{aligned}
$$

Note that for $z=1$, equations (5.44) and (5.33) imply that $M(1)^{a b}$ is a Casimir functional of the canonical Poisson bracket $\theta^{\text {str }}$. This is because at $z=1$ the transfer matrix $P \exp \int_{C} d g g^{-1}$ over the contour $C$ is expressed in terms of the string worldsheet fields at the endpoints of $C$.

This reasoning is rather formal, because we have not taken into account the boundary terms. We have not studied the boundary conditions in this paper. But we expect that with the boundary conditions properly taken into account, only the traces $\operatorname{tr} M(z)$ are Casimir functionals. This would imply that the traces of the monodromy matrix are in involution with respect to all the compatible Poisson brackets, see the last page of [41].

\section{Geometrical meaning of the boost-invariant Poisson bracket}

In this section we will give a geometrical description of the boost-invariant Poisson bracket in the special case when the motion of the string is restricted to $\mathbf{R} \times S^{N}$.

The classical string is equivalent to the non-linear sigma-model (3.5) with the Virasoro constraints (3.4). As we discussed in Section 2.3, through each point of the string worldsheet pass two lightlike curves $C^{+}$and $C^{-}$called 
characteristics. In this section we will consider the projections of $C^{+}$and $C^{-}$to $S^{5}$, and also denote them $C^{+}$and $C^{-}$. The discussion in this section applies to $S^{N}$ for arbitrary $N$.

Let $K^{+}$and $K^{-}$be the unit vectors on the worldsheet orthogonal to $C^{+}$ and $C^{-}$respectively.
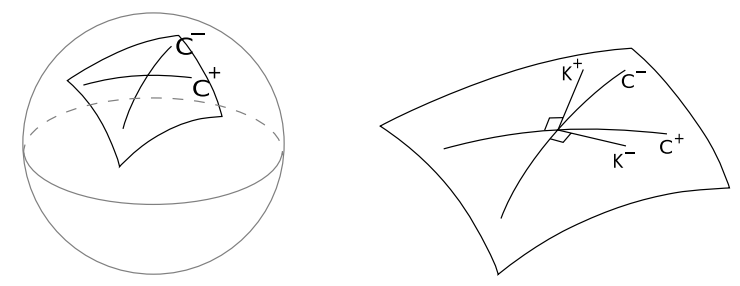

Consider also the normal bundle $\mathcal{N}$ to $\Sigma$ in $S^{N}$. It consists of those vectors in $T S^{N}$ which are orthogonal to $T \Sigma$. The rank of $\mathcal{N}$ is $N-2$, for example for $S^{N}=S^{5}$ we get three normal vectors at each point of $\Sigma$. Let us consider the vector bundles $\mathcal{N} \oplus K^{+}$and $\mathcal{N} \oplus K^{-}$, both having the rank $N-1$.
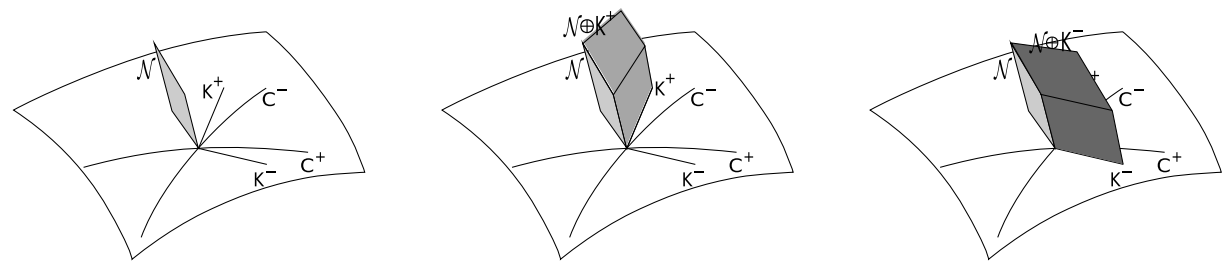

Let us restrict the standard Levi-Civita connection to $\mathcal{N} \oplus K^{+}$and $\mathcal{N} \oplus$ $K^{-}$. We will denote the restricted connections $\nabla^{L}$ and $\nabla^{R}$ :

$$
\begin{aligned}
\nabla^{L} & =\left.D_{\overline{0}}\right|_{\mathcal{N} \oplus K^{+}}, \\
\nabla^{R} & =\left.D_{\overline{0}}\right|_{\mathcal{N} \oplus K^{-}} .
\end{aligned}
$$

"Restricted connection" means that, for example,

$$
\nabla^{L} v=\mathcal{P}_{\mathcal{N} \oplus K^{+}} D_{\overline{0}} v .
$$

where $v$ is a section of $\mathcal{N} \oplus K^{+}$and $\mathcal{P}_{\mathcal{N} \oplus K^{+}}$is the projection on $\mathcal{N} \oplus K^{+} \subset$ $T S^{N}$. It is easy to verify that both $\nabla^{L}$ and $\nabla^{R}$ are flat connections:

$$
\left[\nabla_{+}^{L}, \nabla_{-}^{L}\right]=0, \quad\left[\nabla_{+}^{R}, \nabla_{-}^{R}\right]=0 .
$$

This follows from the string worldsheet equations of motion. 
Let us introduce some trivialization of $\mathcal{N}$. A trivialization is a choice of $N-2$ sections $\mathbf{e}_{1}, \ldots, \mathbf{e}_{N-2}$ of $\mathcal{N}$ which form an orthonormal system:

$$
\left(\mathbf{e}_{i}, \mathbf{e}_{j}\right)=\delta_{i j} .
$$

Note that the trivialization of $\mathcal{N}$ defines the trivializations of both $\mathcal{N} \oplus K^{+}$ and $\mathcal{N} \oplus K^{-}$. Indeed, to get an orthonormal system in $\mathcal{N} \oplus K^{+}$we just add to $\mathbf{e}_{1}, \ldots, \mathbf{e}_{N-2}$ the unit vector in $K^{+}$.

Having the trivializations $\mathcal{N} \oplus K^{+} \simeq \mathbf{R}^{N-1}$ and $\mathcal{N} \oplus K^{-} \simeq \mathbf{R}^{N-1}$ we can consider the monodromies of $\nabla^{L}$ and $\nabla^{R}$. The monodromies are the orthogonal matrices $g^{L}$ and $g^{R}$ satisfying the equations:

$$
\nabla^{L} g^{L}=0, \quad \nabla^{R} g^{R}=0 .
$$

With these notations, the boost-invariant symplectic structure is given by the following formula:

$$
\begin{aligned}
\Omega= & \oint[8 \delta \varphi * d \delta \varphi \\
& \left.+\operatorname{tr}\left(\left(\delta g_{L} g_{L}^{-1}\right) \delta\left(d g_{L} g_{L}^{-1}\right)\right)-\operatorname{tr}\left(\left(\delta g_{R} g_{R}^{-1}\right) \delta\left(d g_{R} g_{R}^{-1}\right)\right)\right],
\end{aligned}
$$

where $2 \varphi$ is the angle between $C^{+}$and $C^{-}$. One can verify by an explicit calculation that (6.4) does not depend on the choice of the contour, and on the choice of the trivialization of $\mathcal{N}$. To prove that equation (6.4) is equivalent to equation (5.18) we note that $f$ is related to $g_{L}$ and $g_{R}$ in the following way:

$$
f=\left[\begin{array}{cc}
1 & \mathbf{0} \\
\mathbf{0} & g_{R}^{-1}
\end{array}\right]\left[\begin{array}{ccc}
\cos 2 \varphi & -\sin 2 \varphi & \mathbf{0} \\
\sin 2 \varphi & \cos 2 \varphi & \mathbf{0} \\
\mathbf{0} & \mathbf{0} & \mathbf{1}
\end{array}\right]\left[\begin{array}{cc}
1 & \mathbf{0} \\
\mathbf{0} & g_{L}
\end{array}\right]
$$

Let us first choose a gauge so that

$$
J_{\overline{2}-}=\left(\begin{array}{lll}
0 & 1 & 0_{1 \times(N-1)} \\
-1 & 0 & 0_{1 \times(N-1)} \\
0_{(N-1) \times 1} & 0_{(N-1) \times 1} & 0_{(N-1) \times(N-1)}
\end{array}\right) .
$$

In this gauge

$$
J_{\overline{0}+}=\left(\begin{array}{ll}
0_{2 \times 2} & 0_{2 \times(N-1)} \\
0_{(N-1) \times 2} & -\partial_{+} g_{R} g_{R}^{-1}
\end{array}\right),
$$


because $D_{\overline{0}+} J_{\overline{2}_{-}}=0$. Now let us switch to the gauge where

$$
J_{\overline{2}+}=\left(\begin{array}{lll}
0 & 1 & 0_{1 \times(N-1)} \\
-1 & 0 & 0_{1 \times(N-1)} \\
0_{(N-1) \times 1} & 0_{(N-1) \times 1} & 0_{(N-1) \times(N-1)}
\end{array}\right) .
$$

This requires the gauge transformation

$$
\left(\begin{array}{llll}
1 & 0 & 0 & 0_{1 \times(N-2)} \\
0 & \cos 2 \varphi & -\sin 2 \varphi & 0_{1 \times(N-2)} \\
0 & \sin 2 \varphi & \cos 2 \varphi & 0_{1 \times(N-2)} \\
0 & 0_{(N-2) \times 1} & 0_{(N-2) \times 1} & 1_{(N-2) \times(N-2)}
\end{array}\right) .
$$

Finally, the gauge transformation $\left(\begin{array}{ll}1_{2 \times 2} & 0_{2 \times(N-1)} \\ 0_{(N-1) \times 2} & g_{L}\end{array}\right)$ brings us to the normal frame.

Equation (6.5) allows us to prove equation (6.4) using the PolyakovWiegmann type of identities. Let us denote:

$$
f_{R}^{-1}=\left[\begin{array}{cc}
1 & \mathbf{0} \\
\mathbf{0} & g_{R}^{-1}
\end{array}\right], \quad f_{2 \varphi}^{-1}=\left[\begin{array}{ccc}
\cos 2 \varphi & -\sin 2 \varphi & \mathbf{0} \\
\sin 2 \varphi & \cos 2 \varphi & \mathbf{0} \\
\mathbf{0} & \mathbf{0} & \mathbf{1}
\end{array}\right], \quad f_{L}=\left[\begin{array}{cc}
1 & \mathbf{0} \\
\mathbf{0} & g_{L}
\end{array}\right] .
$$

We now have $f=f_{R}^{-1} f_{2 \varphi}^{-1} f_{L}$. Using the fact that $d f f^{-1}$ is of the form

$$
d f f^{-1}=\left[\begin{array}{ccccc}
0 & * & * & * & * \\
* & 0 & 0 & 0 & 0 \\
* & 0 & 0 & 0 & 0 \\
* & 0 & 0 & 0 & 0 \\
* & 0 & 0 & 0 & 0
\end{array}\right]
$$

we can show that

$$
\begin{aligned}
\operatorname{tr} & \left(\delta\left(f_{R} f\right)\left(f_{R} f\right)^{-1} \delta\left(\partial_{+}\left(f_{R} f\right)\left(f_{R} f\right)^{-1}\right)\right. \\
= & \operatorname{tr}\left(\delta f_{R} f^{-1} \delta\left(\partial_{+} f_{R} f_{R}^{-1}\right)\right)+\operatorname{tr}\left(\delta f f^{-1} \delta\left(\partial_{+} f f^{-1}\right)\right) \\
& +\partial_{+} \operatorname{tr}\left(\delta f f^{-1} f_{R}^{-1} \delta f_{R}\right) .
\end{aligned}
$$

On the other hand this is equal to:

$$
\begin{aligned}
\operatorname{tr}\left(\delta\left(f_{2 \varphi}^{-1} f_{L}\right) f_{L}^{-1} f_{2 \varphi} \delta\left(\partial_{+}\left(f_{2 \varphi}^{-1} f_{L}\right) f_{L}^{-1} f_{2 \varphi}\right)\right)= & \operatorname{tr}\left(f_{2 \varphi}^{-1} \delta f_{2 \varphi} \delta\left(f_{2 \varphi}^{-1} \partial_{+} f_{2 \varphi}\right)\right) \\
& +\operatorname{tr}\left(\delta f_{L} f_{L}^{-1} \delta\left(\partial_{+} f_{L} f_{L}^{-1}\right)\right)
\end{aligned}
$$


This equation and equation (6.6) imply that the symplectic structure (6.4) is on the light cone equal to the first term in (5.23):

$$
\int d \tau^{+} \operatorname{tr}\left(\delta f f^{-1} \delta\left(\partial_{+} f f^{-1}\right)\right) .
$$

It would be interesting to find a similar geometrical interpretation for the symplectic structure (5.23) of the full superstring in $A d S_{5} \times S^{5}$.

\section{Acknowledgments}

I would like to thank N. J. Berkovits for discussions of the zero curvature equations and dressing transformations, S. Cherkis and A. Tseytlin for comments, and Google Scholar for pointing me to the references [36] and [6-15]. This research was supported by the Sherman Fairchild Fellowship and in part by the RFBR Grant No. 06-02-17383 and in part by the Russian Grant for the support of the scientific schools NSh-1999.2003.2.

\section{References}

[1] A. A. Tseytlin, Spinning strings and AdS/CFT duality, arXiv:hep-th/0311139.

[2] K. Zarembo, Semiclassical Bethe ansatz and AdS/CFT, C. R. Phys. 5 (2004), 1081-1090 [arXiv:hep-th/0411191].

[3] N. Beisert, The dilatation operator of $N=4$ super YangMills theory and integrability, Phys. Rep. 405 (2005), 1-202 [arXiv:hep-th/0407277].

[4] J. Plefka, Spinning strings and integrable spin chains in the AdS/CFT correspondence, arXiv:hep-th/0507136.

[5] K. Pohlmeyer, Integrable hamiltonian systems and interactions through quadratic constraints, Commun. Math. Phys. 46 (1976), 207-221.

[6] A. Doliwa and P. M. Santini, An elementary geometric characterization of the integrable motions of a curve, Phys. Lett. A185 (1994), 373-384.

[7] J. Langer, Recursion in curve geometry, N Y J. Math. 5 (1999), 25-51 (electronic).

[8] J. Langer and R. Perline, Poisson geometry of the filament equation, J. Nonlinear Sci. 1(1) (1991), 71-93.

[9] G. Marí Beffa, J. A. Sanders and J. P. Wang, Integrable systems in threedimensional Riemannian geometry, J. Nonlinear Sci. 12(2) (2002), 143-167. 
[10] J. A. Sanders and J. P. Wang, Integrable systems in n-dimensional Riemannian geometry, Mosc. Math. J. 3(4) (2003), 1369-1393.

[11] G. Marí Beffa, Poisson brackets associated to invariant evolutions of Riemannian curves, Pacific J. Math. 215(2) (2004), 357-380.

[12] G. Marí Beffa, Poisson brackets associated to the conformal geometry of curves, Trans. Amer. Math. Soc. 357(7) (2005), 2799-2827 (electronic).

[13] G. Marí Beffa, Poisson geometry of differential invariants of curves in some nonsemisimple homogeneous spaces, Proc. Amer. Math. Soc. 134(3) (2006), 779-791 (electronic).

[14] S. C. Anco, Bi-Hamiltonian operators, integrable flows of curves using moving frames, and geometric map equations, J. Phys. A: Math. Gen. 39 (2006), 2043-2072, [arXiv:nlin.SI/0512051].

[15] S. C. Anco, Hamiltonian flows of curves in $G / S O(N)$ and vector soliton equations of $m K d V$ and sine-Gordon type, SIGMA 2044 (2006) [arXiv:nlin.SI/0512046].

[16] A. Mikhailov, A nonlocal Poisson bracket of the sine-Gordon model, arXiv:hep-th/0511069.

[17] N. Dorey and B. Vicedo, A symplectic structure for string theory on integrable backgrounds, arXiv:hep-th/0606287.

[18] A. Das, J. Maharana, A. Melikyan and M. Sato, The algebra of transition matrices for the AdS(5) $x S^{* *} 5$ superstring, J. High Energy Phys. 12 (2004), 055 [arXiv:hep-th/0411200].

[19] A. Das, A. Melikyan and M. Sato, The algebra of flat currents for the string on $A d S(5) x S^{* *} 5$ in the light-cone gauge, J. High Energy Phys. 11 (2005), 015 [arXiv:hep-th/0508183].

[20] A. K. Das, Light front Hamiltonian structures for the nonlinear sigma model, Mod. Phys. Lett. A13 (1998), 1133-1142.

[21] M. Bianchi and J. Kluson, Current algebra of the pure spinor superstring in $A d S(5) x S(5)$, arXiv:hep-th/0606188.

[22] D. Bak and S.-J. Rey, Holographic view of causality and locality via branes in AdS/CFT correspondence, Nucl. Phys. B572 (2000), 151187 [arXiv: hep-th/9902101].

[23] J. Callan, Curtis G. and A. Guijosa, Undulating strings and gauge theory waves, Nucl. Phys. B565 (2000), 157-175 [arXiv:hep-th/9906153].

[24] A. Mikhailov, Nonlinear waves in AdS/CFT correspondence, arXiv:hep-th/0305196.

[25] G. Falqui, Poisson pencils, integrability, and separation of variables, arXiv:nlin.SI/0310028. 
[26] A. Sergyeyev, A simple way of making a Hamiltonian system into a bi-Hamiltonian one, Acta Appl. Math. 83(1-2) (2004), 183-197.

[27] C. Klimčík and P. Ševera, Dual non-abelian duality and the Drinfeld double, Phys. Lett. B351(4) (1995), 455-462.

[28] S. Klimchik and S. E. Parkhomenko, On the Poisson-Lie T-duality and zero modes, Teoret. Mat. Fiz. 139(3) (2004), 462-476.

[29] R. R. Metsaev and A. A. Tseytlin, Type IIB superstring action in AdS(5) x S(5) background, Nucl. Phys. B533 (1998), 109-126 [arXiv:hep-th/9805028].

[30] R. Roiban and W. Siegel, Superstrings on $A d S(5) \times S(5)$ supertwistor space, J. High Energy Phys. 11 (2000), 024 [arXiv:hep-th/0010104].

[31] I. Bena, J. Polchinski and R. Roiban, Hidden symmetries of the AdS(5) $x S^{* * 5}$ superstring, Phys. Rev. D69 (2004), 046002 [arXiv:hep-th/0305116].

[32] N. Beisert, V. A. Kazakov, K. Sakai and K. Zarembo, The algebraic curve of classical superstrings on $\operatorname{AdS(5)} x S^{* * 5}$, arXiv:hep-th/0502226.

[33] C. Bår, Real killing spinors and holonomy, Commun. Math. Phys. 154 (1993), 509521.

[34] A. Mikhailov, Giant gravitons from holomorphic surfaces, J. High Energy Phys. 11 (2000), 027 [arXiv:hep-th/0010206].

[35] A. Mikhailov, Supersymmetric null-surfaces, J. High Energy Phys. 09 (2004), 068 [arXiv:hep-th/0404173].

[36] I. Bakas, Q.-H. Park and H.-J. Shin, Lagrangian formulation of symmetric space sine-Gordon models, Phys. Lett. B372 (1996), 45-52 [arXiv:hep-th/9512030].

[37] E. Witten, Nonabelian bosonization in two dimensions, Commun. Math. Phys. 92 (1984), 455-472.

[38] R. L. Bishop, There is more than one way to frame a curve, Amer. Math. Monthly 82 (1975), 246-251.

[39] A. M. Polyakov, Supermagnets and sigma models, arXiv:hep-th/0512310.

[40] E. V. Ferapontov, Restriction, in the sense of Dirac, of the Hamiltonian operator $\delta^{I J}(d / d x)$ to a surface of the Euclidean space with a plane normal connection, Funktsional. Anal. i Prilozhen., 26(4), 1992, pp. 83-86.

[41] L. D. Faddeev and L. A. Takhtajan, Hamiltonian methods in the theory of solitons. Translated by A. G. Reyman, Springer-Verlag, Berlin, Heidelberg and New York, 1987, pp. ix + 592. 